\title{
1 Prediction of wave ripple characteristics using genetic programming
}

2 Evan B. Goldstein*1 ${ }^{1}$, Giovanni Coco $^{2}$, A. Brad Murray ${ }^{1}$

$3 \quad{ }^{1}$ Division of Earth and Ocean Sciences, Nicholas School of the Environment; Center for

4 Nonlinear and Complex Systems, Duke University, Box 90227, Durham, NC 27708

$5 \quad{ }^{2}$ Environmental Hydraulics Institute, "IH Cantabria”, c /Isabel Torres n ${ }^{\circ} 15$., Universidad

6 de Cantabria, Santander, Spain 39011.

$7 \quad *$ corresponding author: evan.goldstein@unc.edu

9 Keywords: Ripples; Bedforms; Genetic programming; Machine learning; Data driven

10 Prediction; Symbolic regression

11

12 Cite as: E. B. Goldstein, G. Coco, A. B. Murray, 2013. Prediction of wave ripple

13 characteristics using genetic programming, Continental Shelf Research, V. 71, p.1-15,

14 https://doi.org/10.1016/j.csr.2013.09.020. 


\section{Abstract}

17 We integrate published data sets of field and laboratory experiments of wave

18 ripples and use genetic programming, a machine learning paradigm, in an attempt to

19 develop a universal equilibrium predictor for ripple wavelength, height, and steepness.

20 We train our genetic programming algorithm with data selected using a maximum

21 dissimilarity selection routine. Thanks to this selection algorithm we use less data to train

22 the genetic programming software, allowing more data to be used as testing (i.e. to

23 compare our predictor vs. common prediction schemes). Our resulting predictor is

24 smooth and physically meaningful, different from other machine learning derived results.

25 Furthermore our predictor incorporates wave orbital ripples that were previously

26 excluded from empirical prediction schemes, notably ripples in coarse sediment and long

27 wavelength, low height ripples ('hummocks'). This new predictor shows ripple length to

28 be a weakly nonlinear function of both bottom orbital excursion and grain size. Ripple

29 height and steepness are both nonlinear functions of grain size and predicted ripple length

30 (i.e. bottom orbital excursion and grain size). We test this new prediction scheme against

31 common (and recent) predictors and the new predictors yield a lower normalized root

32 mean squared error using the testing data. This study further demonstrates the

33 applicability of machine learning techniques to successfully develop well performing

34 predictors if data sets are large in size, extensive in scope, multidimensional, and

35 nonlinear.

37 1. Introduction 
Sufficiently strong water wave propagation over a moveable bed composed of sand

39 grains results in the development rhythmic bedforms whose crest spacing is of the order

40 of centimeters to meters while heights are of the order of centimeters. These features are

41 often termed vortex ripples because of a recirculation cell that develops on the lee side of

42 the bedform that is subsequently ejected upward during reversals in flow direction.

43 Accurate prediction of vortex ripple size and shape is crucial for successful determination

44 of seabed bottom roughness, a first order control on wave attenuation (e.g., Ardhuin et

45 al., 2002), as well as sediment transport as suspended load (e.g., Green and Black, 1999;

46 Bolaños et al., 2012). Furthermore ripple migration is a fundamental mechanism of

47 bedload transport (e.g., Traykovski et al 1999; Becker et al., 2007), and parameterizations

48 of bedload flux necessitate an accurate depiction of ripple size and shape.

49 Many predictors of equilibrium ripple geometry have been developed from field

50 and laboratory datasets (e.g. Clifton, 1976; Nielsen, 1981; Grant and Madsen, 1982;

51 Wiberg and Harris, 1994; Faraci and Foti, 2002; Styles and Glenn, 2002; Grasmeijer and

52 Kleinhans, 2004; Soulsby and Whitehouse, 2005; Soulsby et al., 2012; Pedocchi and

53 García, 2009a; Camenen, 2009). Equilibrium ripple size and shape is frequently broken

54 down to include 3 subpopulations, a convention developed by Clifton (1976), and

55 reviewed here in order of increasing hydrodynamic forcing. Orbital ripples are believed

56 to scale linearly with wave orbital diameter at the seabed and display the largest steepness

57 (ripple height/wavelength $\sim 0.15$ ). Suborbital ripples show spacing that depends on wave

58 orbital diameter and grain size. In even stronger hydrodynamic conditions anorbital

59 ripples form, whose size is related to grain size alone and whose scaling is irrespective of

60 wave orbital diameter. Suborbital ripples link the population of anorbital ripples with 
61 those of orbital ripples.

62 As noted by Smith and Wiberg (2006), recent field and laboratory work has

63 challenged the existing typology for wave-generated ripples as a result of the addition of

64 two new populations (Figure 1). The first are ripples measured in fine sand under strong

65 hydrodynamic conditions. Field and laboratory campaigns in more energetic conditions

66 have discovered the presence of long wavelength, low amplitude ripples ('hummocks') in

67 fine sands that scale with orbital diameter (e.g. Hanes et al., 2001, O’Donoghue et al.,

68 2006). Predictors are unable to accurately capture this ripple size and shape (e.g. Bolaños

69 et al., 2012), yet modeling (Chang and Hanes, 2004) and observation (Green and Black,

70 1999; Cummings et al., 2009) of these bedforms show they eject vortices and are

71 therefore important for their influence on seabed roughness and sediment transport.

72 Furthermore at times these long wavelength ripples have superimposed anorbital ripples

73 (e.g. Southard et al., 1990; Hanes et al., 2001; Williams et al., 2004), another unsolved

74 problem in wave ripple prediction. Because of these complications, Pedocchi and García

75 (2009a), who developed a recent well performing predictor, omit long wavelength ripples

76 from their analysis, but note that these long wavelength 'round crested' ripples are

77 observed above a critical threshold in $\mathrm{U} / \mathrm{w}_{\mathrm{s}}$ (where $\mathrm{U}$ is the maximum orbital velocity at

78 the bed and $\mathrm{w}_{\mathrm{s}}$ is the sediment fall velocity). Dumas et al. (2005) and Cummings et al.

79 (2009) also show that the transition from anorbital scale ripples to round crested long

80 wave orbital scale ripples is a function of orbital velocity (a set value for their given

81 sediment mixtures).

82 The second new population of ripples are those found in medium to coarse sand

83 (Traykovski et al., 1999; Ardhuin et al., 2002; Becker et al., 2007; Masselinsk et al., 
84 2007; Traykovski, 2007; Cummings et al., 2009; Yamaguchi and Sekiguchi, 2011).

85 Coarse grained ripples have been observed in shelf environments for several decades

86 (e.g., Forbes and Boyd, 1987; Leckie et al., 1988 and references therein) but until

87 recently ripple measurements have not been coupled to the hydrodynamic parameters of

88 their formation. Recent lab work by Cummings et al., (2009) demonstrated the

89 persistence of steep ripples with orbital scaling in coarse sand under strong hydrodynamic

90 conditions.

91 These two new populations of ripples highlight a perennial problem with empirical

92 predictors; unless equations are built using large, integrated data sets that encompass

93 many conditions, prediction schemes are difficult to translate to different settings. A non-

94 empirical approach, such as models based on first principles (e.g., Foti and Blondeaux,

95 1995; Blondeaux, 2001; Charru and Hinch, 2006), presents different problems: nonlinear,

96 emergent processes that occur at the ripple scale such as flow separation, vortex ejection,

97 turbulence, sediment suspension, pattern coarsening, defect creation, migration and

98 annihilation (Werner and Kocurek, 1999), and the existence of multiple stable

99 configurations in ripple sizes/shapes at a given hydrodynamic condition (a stability

100 balloon; Hansen et al., 2001) limit the usefulness of finite-amplitude predictions.

101 Prediction by numerical models of coupled fluid flow and bed evolution present

102 promising results but have so far been tested under a narrow range of conditions and

103 compared to few data sets (Marieu et al., 2008; Chou and Fringer, 2010).

104 If empirical data driven predictors are currently the most broadly applicable tools to

105 develop field scale predictions, how should they be built? Traditionally the development

106 of an empirical predictor relies on transforming a single (or several) noisy 
107 multidimensional dataset to lower-dimensions and fitting a curve (with a set functional

108 form) through the resultant point cloud. Here we offer a different solution: a data

109 integration campaign (the collection of many published datasets) followed by machine

110 learning (ML), whereby computational optimization techniques are used to find solutions

111 to multidimensional and nonlinear problems. The suite of techniques encompassed by

112 ML are essentially identical to empirical data driven techniques used previously except

113 the trial and optimization of solutions is outsourced to a computer.

114 The most common ML paradigm used in coastal studies is artificial neural networks

115 (ANN). Recent examples of its use include predictions of alongshore sediment transport

116 in the surfzone (van Maanen et al., 2010), sand bar behavior (Pape et al., 2010) and

117 suspended sediment reference concentration under waves (Oehler et al., 2012). Yan et al.

118 (2008) used an artificial neural network to predict wave ripple geometry (length and

119 height) based on three input parameters (median grain size, wave period, and the

120 maximum near bed wave orbital velocity). ANN results give better predictions based on 3

121 statistical measures (scatter index, correlation coefficient, and mean geometric deviation)

122 than four common empirical models (Nielsen, 1981; Van Rijn, 1993; Wiberg and Harris,

123 1994; Grasmeijer and Kleinhans, 2004). Yet the ANN ripple prediction scheme derived

124 by Yan et al. (2008) was developed and compared to a limited dataset. Furthermore

125 ANNs are problematic because the highly nonlinear result is difficult to interpret and

126 does not offer immediate insight into the physical nature of the problem at hand. Decision

127 or regression trees (e.g., Oehler et al., 2012), another common and well performing ML

128 technique, is also hampered by the lack of direct physical significance and other

129 drawbacks such as the lack of smoothness. 
In this contribution we use genetic programming (GP; Koza, 1992), a population

131 based optimization technique where the population consists of individual equations (i.e. a

132 population of individual predictors). The mathematical or logical operations that

133 constitute each algorithms can be modified at every time step via an 'evolutionary'

134 process (such as crossover and mutation) to produce expressions that optimize model-

135 data fit. Outputs developed by GP can be smooth functions that are easy to examine and

136 interpret for physical significance. Furthermore, a priori determination of the functional

137 form of the predictor is not required and the final optimized solution can take on any

138 mathematical form (within user defined limits). Thus far genetic programming has been

139 applied to a wide range of problems including the prediction of freshwater phytoplankton

140 dynamics (Whigam and Recknagel, 1999), downscaling of atmospheric model output

141 (Coulibaly, 2004), determining appropriate parameterization for roughness in vegetated

142 flows (Baptist et al., 2007), wave forecasting (Kambekar and Deo, 2012) and mapping of

143 seafloor habitats (Silva and Tseng, 2008).

144 The goal of this study is to demonstrate the applicability of ML techniques

145 (specifically GP) to research questions in the coastal domain. To accomplish this goal we

146 compile 27 different field and laboratory data sets of wave ripple prediction (995

147 individual measurements; Table 1) that span a broad range of conditions and develop a

148 new wave-ripple predictor that is able to capture the morphology of ripple geometry in a

149 wide range of forcing conditions, including conditions where long wave orbital ripples

150 are present. We put our results in the context of existing formulations and theories, and

151 assess the physical relevance of GP predictors. Our new equilibrium predictor ignores the

152 effect of ripple orientation, time evolution, heterogeneous sediment, superimposed 
153 current, ripple asymmetry, and bio-degradation of ripples. We discuss these limitations in

154 the discussion section but note here that other existing time dependent ripple prediction

155 schemes capture one or more (but not all) of these processes (i.e., Soulsby et al., 2012;

156 Traykovski 2007). Finally, the compilation of published ripple data allows for the

157 identification of gaps in knowledge and observations that should be pursued in future

158 research. Future data collection campaigns can be added to this database, allowing for

159 modifications to the prediction schemes shown below. In this sense the ripple prediction

160 scheme we demonstrate here is dynamic.

161

162 2. Data

163 As a result of decades of study, many wave ripple datasets are available in the

164 scientific literature. Examples of recent wave ripple data integration and compilations are

165 Soulsby and Whitehouse (2005), Pedocchi and García (2009a) and Camenen (2009).

166 Here we follow the lead of Pedocchi and García (2009a) and limit our data collection to

167 studies using sediment with quartz (or near quartz) densities $\left(2.65 \mathrm{~g} / \mathrm{cm}^{3}\right)$ performed in

168 large oscillatory tunnels, large wave flumes, wave racetracks and field conditions (i.e. we

169 omit oscillating trays). Data on rolling-grain ripples, small bedforms that initially appear

170 when flat beds are subject to oscillatory water motion, are ignored in this study because

171 they have been experimentally shown to be a transient stage of ripple evolution (Faraci

172 and Foti, 2001). We use 27 published studies in our dataset. Each measurement contains

173 wave ripple, hydrodynamic, and sedimentological parameters. The dataset is split 59\% /

$17441 \%$ between laboratory and field conditions (Table 1), and laboratory measurements are

175 obtained from a 49\% / 49\% / 2\% split between oscillatory tunnels, wave flumes, and 
176 wave racetracks. Measurement error is different for each data set in our database, a

177 natural consequence of data integration campaigns that assemble data collected by

178 different instruments and techniques. We assume that measurements of ripple data

179 obtained in field settings are at or near equilibrium.

180 Our database can be visualized as a series of histograms showing the parameter

181 range in our dataset (Figure 2). A majority of ripple measurements in our database occur

182 at hydrodynamic conditions of $\mathrm{d}_{0}<2 \mathrm{~m}, \mathrm{U}<0.75 \mathrm{~m} / \mathrm{s}$ and sedimentological conditions of

$183 \mathrm{D}_{50}<0.5 \mathrm{~mm}$. Another notable attribute is the strong bimodal signature of ripple

184 steepness centered at values of $\sim 0.15$ and $\sim 0.01$. These clusters represent steep ripples

185 and 'hummocky' ripples, respectively. We base our prediction of wave ripple wavelength

$186 \lambda(\mathrm{m})$, ripple height $\eta(\mathrm{m})$, and ripple steepness $\vartheta(\eta / \lambda$; dimensionless $)$ on four variables:

187 wave period $\mathrm{T}(\mathrm{s})$, bottom orbital excursion $\mathrm{d}_{0}(\mathrm{~m})$, median grain size $\mathrm{D}_{50}(\mathrm{~m})$, and

188 maximum near bed orbital velocity $\mathrm{U}(\mathrm{m} / \mathrm{s})$. A hallmark of field data sets is the irregular

189 forcing, requiring us to reconcile different measured parameters. Several field datasets

190 used in the compiled dataset reported hydrodynamic parameters in terms of significant

191 values $\left(\mathrm{U}_{\text {sig }}, \mathrm{d}_{0, \mathrm{sig}}\right.$, and $\left.\mathrm{T}_{\text {sig }}\right)$. We followed the protocol of Pedocchi and García (2009) and

192 assume $\mathrm{U}=\mathrm{U}_{\text {sig }}$ (and furthermore $\mathrm{d}_{0}=\mathrm{d}_{0, \text { sig }}$ and $\mathrm{T}=\mathrm{T}_{\text {sig }}$ ). We acknowledge that the

193 merging of disparate data sources introduces uncertainty into the data.

194 The hydrodynamic and sedimentological conditions covered by this dataset can be

195 visualized using 6 projections of the 4 dimensional phase space (Figure 3). Notable

196 sparseness occurs in this database at strong hydrodynamic conditions, and at median

197 seabed grain sizes above $0.5 \mathrm{~mm}$. We use $\mathrm{T}, \mathrm{d}_{0}$, and $\mathrm{U}$ as separate independent variables

198 for input to the GP (though they are related by $\mathrm{d}_{0}=\mathrm{UT} / \pi$ ) in an attempt to introduce no 
199 additional information about which of these parameters is most relevant. As GP is a data

200 driven technique, the raw hydrodynamic data is given as input and the ML process

201 determines which hydrodynamic variable(s) is most relevant from a statistical standpoint.

202 We use $\mathrm{T}, \mathrm{d}_{0}, \mathrm{D}_{50}$, and $\mathrm{U}$ to predict $\lambda$. Predicted $\lambda$ is incorporated into the suite of

203 variables (i.e., $T, d_{0}, D_{50}, U$ ) used to predict $\eta$. We combining the predictors for $\lambda$ and $\eta$

204 enable the development of a predictor for ripple steepness. Yet we do not enforce the

205 accurate depiction of steepness in the development of ripple height and length predictors

206 and imprecision in the $\lambda$ and $\eta$ equations may cause imprecision in the prediction of $\vartheta$.

207 However, in some circumstances accurate depiction of height and steepness is required

208 for the parameterization of relevant processes (e.g. vertical suspended sediment

209 diffusivity; Nielsen, 1992): therefore we also develop an independent ripple steepness

210 predictor using the genetic programming technique. The development of a third predictor

211 also further demonstrates the strengths and weaknesses of GP and ML techniques.

212 Predicted $\lambda$ and $\eta$ are added to the variables $\left(T, d_{0}, D_{50}, U\right)$ used to predict $\vartheta$. The

213 development of predictors for $\lambda, \eta$ and $\vartheta$ without enforcing interoperability relies on users

214 to decide which predictors are most important for the specific research question.

215 Several published studies measure two superimposed ripple scales (larger orbital

216 scale ripples and smaller anorbital scale ripples) at a single hydrodynamic condition (e.g.

217 Hanes et al., 2001; Pedocchi and García 2009b; Cummings et al., 2009). Work by

218 Cummings et al., (2009) shows that both pattern modes occur as maximum orbital

219 velocity is increased and the ripple pattern transitions from small scale (anorbital) ripples

220 to large scale orbital ripples ('hummocks'). Upon further velocity increase, the small

221 scale ripples are destroyed and only the large scale orbital features remain (Cummings et 
222 al., 2009). The threshold of large scale orbital ripple appearance can be estimated from

223 the work of Pedocchi and García (2009a) who found that large scale features appear at a

224 threshold value of $\mathrm{U} / \mathrm{w}_{\mathrm{s}} \cong 25$. When both anorbital and large scale ripples are present in

225 tabulated data (e.g. Hanes et al 2001) we only include large scale ripples: the scaling of

226 long wave ripples with bottom orbital diameter suggests a physical relationship to small

227 scale orbital and suborbital ripples. In contrast, anorbital ripples scale with grain size

228 (similar to current ripples) and the mechanism responsible for their formation may be

229 different (Wiberg and Harris, 1994). We remove small-scale (anorbital) ripples from our

230 database if they are present at values of $\mathrm{U}_{0} / \mathrm{w}_{\mathrm{s}} \geq 25$; laboratory work by Cummings et al.,

231 (2009) and Pedocchi and García (2009a) suggests that this regime is dominated by large

232 scale ripples. The targeted collection of field and laboratory data is needed to refine this

233 threshold.

235 3. Methods

2363.1 Selection of training, validation, and testing data

237 The database is split into three subsets to be used as training, validation, and

238 testing. The GP algorithm uses the training dataset to develop and optimize candidate

239 solutions. The validation dataset is used to evaluate the fitness of GP derived solutions

240 and define which predictors persist. Testing data is not used or seen by the GP algorithm

241 and is instead reserved as an independent test of the final predictors (and other published

242 predictors). In the genetic programming literature there remains no proven 'best practice'

243 for percentage of training, validation, and testing data, nor a well defined method of

244 splitting these datasets. This may be because data splitting (e.g., the retention of a testing 
245 dataset) is not addressed in the foundational literature of the technique (as noted by

246 Kushchu, 2002). Yet because our database of ripple measurements contains only sparse

247 data at energetic hydrodynamic conditions and large grain sizes, the selection and

248 partitioning of data into these three categories is crucial to develop a well performing

249 predictor applicable to a range of environments (Bowden et al., 2002). For example,

250 random division of the data has the potential to produce a significant problem; the

251 training data is likely to misrepresent the full phase space of the entire dataset (i.e.

252 exclude coarse grained and/or strong hydrodynamic data).

253 Informed data selection (i.e., selection based on clustering) has been shown to

254 produce better results with ML predictors than 'blind' or random data selection (e.g.,

255 Bowden et al., 2002; May et al 2010). In this study we select training data through the use

256 of a maximum dissimilarity algorithm (MDA; e.g., Camus et al., 2011). This algorithm is

257 not a clustering routine (where cluster centroids are selected to represent a representative

258 value of the data in the cluster), but instead a selection routine (where a centroid

259 represents the most dissimilar data point from the previous centroids; Camus et al., 2011).

260 Though our selection technique is different than the clustering techniques used by

261 Bowden et al. (2002), our approach leads to a similar result: the use of a minimum of

262 training data that is able to capture the variance in hydrodynamic and sedimentological

263 conditions of the entire dataset while leaving more data to be used as validation and

264 testing.

265 Our implemented version of the maximum dissimilarity algorithm is based on the

266 description provided in Camus et al. (2011). Selection starts with the normalization of the

267 data to a value between 0 and 1 : 


$$
X_{n}=\frac{X-X_{\min }}{X_{\max }-X_{\min }}
$$

268 where $X_{n}$ is the new normalized data value (between 0 and 1), $X$ is the original value,

$269 \mathrm{X}_{\min }$ and $\mathrm{X}_{\max }$ are the minimum and maximum of all values of variable $\mathrm{X}$, respectively.

270 After this normalization a single data point, a 'seed', is selected as the first centroid.

271 Since our dataset is typified by sparseness in the coarse grain data, we use the largest

272 grain size measurements as the first centroid (the 'seed'). The user selects the number of

273 centroids and the algorithm then selects the additional centroids through an iterative

274 process: Each data point in our data set is a 4-dimensional vector (normalized T, $\mathrm{U}, \mathrm{d}_{0}$,

$275 \mathrm{D}_{50}$ space) and is associated with a distance to the nearest centroid. The single data point

276 with the maximum distance between itself and the nearest centroid is selected as the next

277 centroid (Camus et al., 2011). This routine continues until the user defined number of

278 centroids is reached, after which data is denormalized.

279 There remains significant ambiguity in determining the appropriate number of

280 centroids (or clusters) needed to accurately represent data, especially continuous data

281 (e.g. May et al 2010). Our dataset on wave ripples is multidimensional and relatively

282 continuous (i.e. not naturally clustered). Furthermore the dataset is sparse in areas

283 because of a lack of collected data, while densely populated with measurements in other

284 regions of phase space (e.g., experimental campaigns at specific hydrodynamic and/or

285 sedimentological conditions). Since we intend to use selected centroids as representatives

286 of the entire dataset, selecting too many centroids will likely rob the validation and

287 testing datasets of poorly represented data (e.g., large $T, U, d_{0}, D_{50}$ ) while too few

288 centroids will leave the testing data with to few data to capture the variability in the

289 dataset. We use 30 centroids for the prediction of $\lambda$ and 40 centroids for the prediction of 
$290 \eta$. Centroids used to represent $\eta$ are also used for analysis of $\vartheta$. Centroid locations can be

291 seen in Figure 4. The use of fewer centroids (10-20) produced too few predictors while

292 more centroids $(\sim 100)$ tended to produce many more nonlinear and potentially overfit

293 solutions. In addition, the solutions obtained with more centroids were qualitatively

294 similar to the solutions presented below using only 30-40 centroids. More centroids are

295 used to predict ripple height because $\eta$ is more difficult to predict (see also Yan et al.,

296 2008; Williams et al., 2004). This is likely a result of the nonlinearities associated with

297 ripple crests protruding into regions of flow with higher velocities: ripple height is likely

298 more strongly influenced by suspension processes as a result. Data selected as the

299 centroid locations are used for the training data. The points not selected as centroids (i.e.

300 not selected as training data) are used for validation and testing data. Data is split

301 between validation and testing randomly, without using a selection routine. Therefore the

302 breakdown for the $\lambda$ datasets is $\sim 3 \%$ training, $\sim 48 \%$ validation, $\sim 48 \%$ testing, while the $\eta$

303 (and $\vartheta$ ) dataset breakdown is $\sim 5 \%$ training, $\sim 47 \%$ validation, $\sim 47 \%$ testing.

304

3053.2 Genetic programming

306 We operate on this compiled ripple data using the evolutionary computation

307 technique of genetic programming (GP), a ML paradigm whereby candidate solutions (in

308 the form of randomly generated equations) are evaluated and subsequently modified

309 (Koza, 1992; Poli et al., 2008). The modification of candidate solutions is manifest as

310 changes in variables and mathematical relationships between variables (i.e. the

311 mathematical form), hence the description of this style of problem as 'symbolic

312 regression'. Variables used in this study to predict wave ripple geometry are $\mathrm{T}, \mathrm{U}, \mathrm{d}_{0}$,

(C) 2017. This manuscript version is made available under the CC-BY-NC-ND 4.0 license http://creativecommons.org/licenses/by-nc-nd/4.0/ 
$313 \mathrm{D}_{50}, \lambda$ (for height and steepness prediction), $\eta$ (for steepness prediction), as well as GP

314 derived constants. Nondimensional, renormalized input (from $0-1$ ) is not necessary with

315 GP (as it is with other ML techniques), and input is fed into the algorithm with units.

316 Only $\mathrm{D}_{50}$ is renormalized in this analysis, and fed into the GP in units of mm (as opposed

317 to $\mathrm{m}$, but the presentation of all results in this contribution are in meters). Mathematical

318 operators used in this study are + (addition), $-($ subtraction $), \times$ (multiplication),$\div$

319 (division), $\sqrt{ }$ (square root), as well as integer powers (e.g. $\left.x^{2}, x^{3}, x^{4}\right)$. Furthermore we

320 omit logical functions (e.g. if-then-else) because of the lack of smoothness when

321 incorporating these components.

322 Candidate solutions are evaluated based a 'fitness function', a user defined error

323 metric that determines how well a given candidate fits the validation data. Mean squared

324 error (MSE) is used as the fitness function:

$$
M S E=\frac{(p-b)^{2}}{n}
$$

325 where MSE is the Mean Squared Error, $\mathrm{n}$ is the sample size, $\mathrm{p}$ are the predicted values,

326 and $b$ are the observed values. The correlation coefficient, one of the error metrics used in

327 previous ripple studies (Yan et al., 2008), was not used as a fitness function because it

328 tended to develop nonphysical predictors (negative wavelengths and heights under certain

329 conditions) that matched the shape of the data but did not align well with actual

330 magnitudes.

331 Equations that minimize mean squared error are retained, while poor performing

332 solutions are discarded. Retained solutions are combined, rearranged and manipulated in

333 a probabilistic manner according to evolutionary processes; solutions 'crossover' by

334 combining elements of other solutions to develop a new solution and 'mutations' develop 
335 new mathematical expression to substitute or tack on to a previous solution. As an

336 example, candidate solutions are commonly encoded in GP software as 'trees', and the

337 modification of candidate solutions (change of variables and/or mathematical expression)

338 is accomplished through adjustments in tree 'limbs' (Figure 5). Through time predictors

339 gain complexity (i.e. trees grow in size) as they are recombined in a variety of ways,

340 moving from simple equations (e.g. two variables and one mathematical symbol linking

341 them) to highly nonlinear, complex expressions (e.g. many variables linked by many

342 symbols). In this way the growth and adjustment of candidate solutions enables the

343 searching of an increasingly larger phase space (i.e. variable and symbolic space), and

344 find optimized solutions to the problem at hand. This search process occurs until a

345 solution with zero error is found or the routine is terminated.

346 In this study we use a proven symbolic regression/genetic programming software

347 package developed by Schmidt and Lipson (2009; 2013). This software package,

348 'Eureqa', modifies the tree-based encoding outlined above by eliminating redundancy

349 when multiple 'tree limbs' are identical. The software output is a suite of solutions with

350 increasing mathematical 'complexity', where complexity is a count of the numbers of

351 operations and variables used in the candidate solution. Each solution of a given

352 complexity represents the equation with the least error compared to identically 'complex'

353 candidate solutions. Furthermore, to be retained in the solution set, a given solutions must

354 have less error compared to all previous less-complex solutions. Therefore the suite of

355 solutions that is developed as output lie along the 'Pareto front', a line in complexity-

356 fitness space that illustrates fitness increases with the increasing complexity of candidate

357 solutions. Because simple predictors are retained though more complex predictors may fit 
358 the data with less error, the user must pick a single solution as the final predictor of

359 choice.

3613.3 Generalization and overfitting

362 The lack of a single optimal solution as output from the GP algorithm is likely a

363 consequence of using noisy data (e.g., field data) and examining a phenomena that may

364 not have a single solution, but instead a small range of possible solutions (i.e., there may

365 be multiple stable ripple configurations for a given hydrodynamic/sedimentological

366 condition, a 'stability balloon'; Hansen et al., 2001). The determination of an ideal

367 solution from the GP program was further complicated because there is no stoppage

368 routine built into the algorithm (e.g., based on fitness) used in this study. We cease the

369 search after roughly $10^{10}$ formulas have been evaluated as continued search shows only

370 marginal increases in predictive power (and this increase occurs only on more complex,

371 likely overfit, predictors). The solutions were then evaluated to determine the most

372 appropriate final predictor. Several methods for eliminating overfit solutions exist (e.g.,

373 Gonçalves et al., 2012). We use several techniques in parallel to determine appropriate

374 solutions: 1) bias toward shorter, physically reasonable solutions, 2) examining 'cliffs' in

375 the Pareto front, and 3) examination of solution fit.

376 Many of the more complex solutions have lower error with training and validation

377 data but are physically uninterpretable. Therefore when evaluating the family of solutions

378 from a given genetic programming iteration we tend to bias our search for the most

379 universal predictor by preferring compact solutions because they tend to offer more

380 generalization and are likely less overfit (The minimum description length principle; e.g., 
381 O'Neill et al., 2010). Shorter solutions reappear with repeat initialization of the genetic

382 programming algorithm, suggesting that these represent the globally optimum solutions

383 for a given function size. Longer solutions do not tend to reappear, either a result of a

384 large search space that is not repeated during repeat initializations or the presence of

385 multiple, equally optimal solutions in the large phase space (i.e. local minima). The

386 inherent reproducibility of simple, weakly nonlinear solutions suggests their use as

387 predictors until further data can be used to justify the use of highly nonlinear predictors.

388 Aside from examining the solutions from least complex to most complex,

389 examining areas along the Pareto front where large gains in prediction are obtained with

390 small gains in solution complexity is a natural place to observe potential solutions (Figure

391 6). These areas along the Pareto front are referred to as 'cliffs'. Schmidt and Lipson

392 (2009) used the last of such 'cliffs' to observe many physically relevant solutions. In this

393 study final solutions were chosen from the subset of solutions that are 'cliffs' along the

394 Pareto front

395 Candidate solutions are evaluated by minimizing error functions. Occasionally

396 candidate solutions are able to minimize the mean squared error but provide unphysical

397 solutions (e.g. negative ripple wavelengths under some conditions) or generally poor

398 global performance (e.g. flat, constant predictors). These solutions must be manually

399 disregarded, as there is as yet no means of excluding them.

400

4013.4 Comparison with other predictors

402 Predictor performance is evaluated, using the independent testing data, with the

403 Normalized Root Mean Squared Error (NRMSE): 


$$
N R M S E=\frac{\sqrt{M S E}}{\bar{b}}
$$

404 where $\bar{b}$ is the mean of the observed values. Additionally we report correlation

405 coefficient (Pearson's $r$ ) for each predictor evaluated against the independent testing data.

406 We compare our results to two recently developed and widely used predictors:

407 Soulsby and Whitehouse (2005; also reported in Soulsby et al., 2012) and Pedocchi and

408 García (2009a). As noted by Soulsby et al., (2012), recent work by Camenen (2009)

409 using a large compiled database of ripple measurements found the Soulsby and

410 Whitehouse (2005) formulation to be the best overall predictor compared to those

411 developed by Grant and Madsen (1982), Wikramanayake and Madsen (1991), Van Rijn

412 (1993), Mogridge et al., (1994), Wiberg and Harris (1994), and Grasmeijer and Kleinhans

413 (2004). The recent work of Pedocchi and García (2009a), which was not evaluated by

414 Camenen (2009), yields good collapse of the data compared to other the predictors

415 mentioned above and performs well in field conditions (Bolaños et al., 2012). The

416 Soulsby and Whitehouse (2005) predictor for length and steepness $(\eta / \lambda)$ is:

$$
\begin{aligned}
& \left.\frac{\lambda}{A}=\left[1+\left(1.87 \times 10^{-3}\right) \frac{A}{D_{50}}\left(1-e^{\left\{-\left(2.0 \times 10^{-4} \frac{A}{D_{50}}\right)^{1.5}\right.}\right\}\right)\right]^{-1} \\
& \left.\frac{\eta}{\lambda}=0.15\left[1-e^{\left\{-\left(5000 \frac{D_{50}}{A}\right)^{3.5}\right.}\right\}\right]
\end{aligned}
$$

417 where $\mathrm{A}$ is the wave orbital amplitude $\left(2 \mathrm{~A}=d_{0}\right)$. Combining (4) and (5) yields $\eta$ alone.

418 The Pedocchi and García (2009a) predictor is: 


$$
\begin{aligned}
& \frac{\lambda}{d_{0}}=\left\{\begin{array}{cc}
0.65\left[\left(0.050 \frac{U}{w_{s}}\right)^{2}+1\right]^{-1}, & R e_{p} \geq 13 \\
0.65\left[\left(0.040 \frac{U}{w_{s}}\right)^{2}+1\right]^{-1}, & 9 \leq R e_{p}<13 \\
0.65\left[\left(0.054 \frac{U}{w_{s}}\right)^{3}+1\right]^{-1}, & R e_{p}<9
\end{array}\right. \\
& \frac{\eta}{d_{0}}=\left\{\begin{array}{cc}
0.1\left[\left(0.055 \frac{U}{w_{s}}\right)^{3}+1\right]^{-1}, & R e_{p} \geq 13 \\
0.1\left[\left(0.055 \frac{U}{w_{s}}\right)^{4}+1\right]^{-1}, & 9 \leq e_{p}<13 \\
0.1\left[\left(0.055 \frac{U}{w_{s}}\right)^{5}+1\right]^{-1}, & R e_{p}<9
\end{array}\right.
\end{aligned}
$$

420 where $\mathrm{w}_{\mathrm{s}}$ is evaluated for $\mathrm{D}_{50}$ and $\mathrm{Re}_{\mathrm{p}}$ is a dimensionless particle size (Pedocchi and

421 García, 2009a) evaluated as:

$$
R e_{p}=\frac{\sqrt{g R D_{50}} D_{50}}{v}
$$

422 where $\mathrm{g}$ is gravity, $\mathrm{R}$ is the submerged specific density of sediment (here taken to be

$4231.65)$ and $v$ is kinematic viscosity. The three size classes $\left(R e_{p} \geq 13,9 \leq R e_{p}<\right.$

42413 and $R e_{p}<9$ ) correspond to coarse, medium and fine sand respectively and the three

425 separate equations result in slight discontinuities.

426 Lastly we note that we are unable to compare the performance of our GP derived

427 predictor to the ANN model developed by Yan et al., (2008) as we do not know the final

428 optimized ANN equation developed by Yan et al., (2008). In addition, we do not know

429 which data was used as training/validation or testing in the development of the ANN

430 model. 


\section{4. Results}

\subsection{Ripple wavelength}

434 The GP algorithm output is shown in Table 2. This experiment evaluated $10^{10}$

435 formulas to develop the Pareto front shown in Figure 6. Cliffs, significant gains in error

436 for small changes in equation complexity occur along the Pareto front at complexities of

4373,6 , and 8 (Figure 6) The first of these cliffs (at complexity 3 ) is a predictor, $\lambda=0.607 \mathrm{~d}_{0}$,

438 that mimics the basic form of the orbital scale (i.e. weak hydrodynamics) predictor

439 commonly used today, where ripple wavelength is a linear function of orbital excursion

440 (e.g. $\lambda=0.65 \mathrm{~d}_{0}$ from Miller and Komar, $1980 \mathrm{a} ; \lambda=0.62 \mathrm{~d}_{0}$ from Wiberg and Harris, 1994).

441 Debate surrounds the correct value of the coefficient modifying orbital excursion,

442 especially in medium to coarse sand (e.g. Becker et al., 2007; Traykovski et al., 1999).

443 All solutions that are more complex than the solution of complexity 3 demonstrate why

444 there is debate: the coefficient is likely a function of grain size. We rule out solution 3 as

445 a viable universal predictor because grain size is a control on ripple length (e.g.,

446 Cummings et al., 2009). We focus our remaining examination on the solution at

447 complexity 8 .

$$
\lambda=\frac{d_{0}}{1.12+2.18\left(1000 D_{50}\right)}
$$

448 Figure 7 shows the general behavior of this predictor: increasing wave ripple

449 spacing with increasing bottom orbital excursion and decreasing wave ripple wavelength

450 with increasing grain size. Furthermore ripple length is more sensitive to median grain

451 size at larger orbital diameter. Previous ripple length prediction schemes have focused on

452 orbital diameter and grain size as they represent the two fundamental length scales in the 
453 development of oscillatory bedform. For instance, Soulsby and Whitehouse (2005)

454 develop an equilibrium predictor where $\mathrm{A} / \mathrm{D}_{50}$ is the controlling parameter after

455 examining the collapse of compiled data with several other variables.

456 Using only the reserved testing data, the NRMSE of the new GP predictor as well

457 as those developed by Soulsby and Whitehouse (2005) and Pedocchi and García (2009a),

458 are $0.74,1.33$, and 1.22 respectively, and the correlation coefficient is $0.78,0.02$, and

4590.20 respectively. The GP derived predictor performs better than the other predictors

460 based on the NRMSE and correlation coefficient. Figure 8 shows the performance of

461 these models in both linear and log-log space. Neither of these previously published

462 predictors were developed for large scale orbital ripples, and both show predictions that

463 deviate significantly when observed ripple wavelengths are large. The GP derived

464 predictor is better able to capture large scale ripples. Both Soulsby and Whitehouse

465 (2005) and Pedocchi and García (2009a) are able to better capture small scale 'anorbital'

466 ripples that deviate significantly from the scaling of (9).

468 4.2 Ripple height

469 The GP algorithm output is shown in Table 3. This experiment evaluated $10^{10}$

470 formulas to develop the Pareto front shown in Figure 9. Cliffs occur along the Pareto

471 front at complexities of 3, 5, 14, 18 and 36 (Figure 9). Predictor of complexity $3, \eta$

$472=0.435 \mathrm{~d}_{0}$, is qualitatively similar to predictions of ripple height in the orbital regime (i.e.

473 weak hydrodynamics) presented in Wiberg and Harris (1994), where ripple wavelength is

474 a function of orbital diameter and ripple steepness $(\eta / \lambda)$ is constant, therefore ripple

475 height is a linear function of bottom orbital diameter. Constant steepness breaks down in 
476 stronger hydrodynamic conditions, and this is reflected in the inclusion of grain size and

477 ripple length in more complex predictors. We have no compelling evidence to use the

478 most nonlinear but best fit solution (36), nor is there compelling evidence at this time that

479 ripple height has a such a strongly nonlinear dependence on grain size (Solution 14 and

480 18). We focus our analysis on solution 5:

$$
\eta=0.313 \lambda\left(1000 D_{50}\right)
$$

481 or, replacing $\lambda$ (which denotes predicted ripple wavelength) with equation 9:

$$
\eta=\frac{0.313 d_{0}\left(1000 D_{50}\right)}{1.12+2.18\left(1000 D_{50}\right)}
$$

Figure 10 shows the behavior of this predictor under conditions of various orbital 483 diameter and grain size. Ripple height increases with increasing grain size and orbital 484 diameter. As with ripple length, ripple height is more sensitive to changes in grain size 485 than changes in orbital velocity. Reserved testing data is used as an independent dataset 486 to compare the GP predictor as well as those developed by Soulsby and Whitehouse 487 (2005) and Pedocchi and García (2009a): the NRMSE for each predictor is 0.79, 1.02, 488 and 1.01 respectively, and the correlation coefficient is $0.67,0.41$, and 0.47 respectively.

489 The GP derived predictor performs better than the other predictors based on the NRMSE 490 and correlation coefficient. Figure 11 shows the performance of these models in both 491 linear and log-log space.

\subsection{Ripple steepness:}

$494 \quad$ Combining the GP predictors for ripple length (9) and height (11), or simply 495 rearranging (10), yields a predictor for ripple steepness: 


$$
\vartheta=0.313\left(1000 D_{50}\right)
$$

496 implying that steepness is a function solely of grain size, which is a gross approximation

497 of the variability observed in the data, and to some extent even unphysical. To enhance

498 our steepness prediction we produce a GP derived steepness predictor. The GP algorithm

499 output is shown in Table 4. This experiment evaluated $10^{10}$ formulas to develop the

500 Pareto front shown in Figure 12. Cliffs, significant gains in error for small changes in

501 complexity occur along the Pareto front at complexities 5, 8, 10, and 16 . The predictor at

502 complexity 5 produces nonphysical results (negative steepness under some conditions) so

503 is ruled out. The most nonlinear predictor reported (complexity of 16) shows only small

504 decrease in error for increasing equation complexity; we focus our analysis on predictor

50510 :

$$
\vartheta=\frac{3.42}{22+\left(\frac{\lambda}{\left(1000 D_{50}\right)}\right)^{2}}
$$

506 by replacing $\lambda$ (predicted ripple wavelength) with (9), yields:

$$
\vartheta=\frac{3.42}{22+\left(\frac{d_{0}}{1.12\left(1000 D_{50}\right)+2.18\left(1000 D_{50}\right)^{2}}\right)^{2}}
$$

507 Figure 13 shows the behavior of this predictor under conditions of various orbital

508 diameter and grain size. Increasing $D_{50}$ (for a given $d_{0}$ ) results in increasing $\vartheta$ until a

509 saturated value of 0.15 is reached. Increasing $d_{0}$ (for a given $D_{50}$ ) results in decreasing $\vartheta$.

510 Small grain sizes are very sensitive to changes in $\mathrm{d}_{0}$, while large grain sizes are relatively

511 insensitive. Figure 14 shows the performance of (14) against the independent testing data

512 compared to the linear convolution of GP derived length and height (12), as well as the

513 Pedocchi and García (2009a) and Soulsby and Whitehouse (2005) predictors. The 
514 NRMSE of these predictors is: $0.36,0.50,0.47$, and 0.43 , respectively, and the

515 correlation coefficient is $0.70,0.48,0.63$, and 0.50 respectively. The GP derived predictor

516 performs better than the other predictors (including the linear convolution of GP derived

$517 \lambda$ and $\eta$ ) based on the NRMSE and correlation coefficient.

519 5. Discussion

5205.1 Predictors derived from genetic programming

521 The suite of predictors that are produced as output of the genetic programming

522 show a trend of increasing predictability with increasing complexity. Highly nonlinear

523 predictors have been avoided in this study because they may be fit to the noise or

524 variance present in the training dataset (i.e. they are overfit). Yet the more complex

525 nonlinear predictors can be used as hypothesis for further field and lab studies where

526 grain size effects are a focus.

527 Dependence on orbital scaling and grain size is not imposed by the authors, it is a

528 result of the data used to feed the genetic programming software. Aside from the data sets

529 used in this study, other field observations have shown decreasing ripple height and

530 increasing ripple length in fine grained sand under strong hydrodynamic forcing (e.g., a

531 transition from steep to low profile bedforms; Green and Black, 1999; Green et al., 2004;

532 Trembanis et al., 2004). Pedocchi and García (2009a) and Cummings et al. (2009) note

533 that $\mathrm{U}$ is the major control on the transition from small ripples (anorbital) to large ripples

534 (hummocks), yet our GP derived predictors contain only one hydrodynamic parameter,

$535 \mathrm{~d}_{0}$. Furthermore dependence on $\mathrm{U}$ is not present in any of the candidate predictors (Tables

5362,3 , and 4). This is likely the result of several factors: First, $d_{0}$ and $U$ are correlated in our 
537 database (Figure 3), making $\mathrm{d}_{0}$ a potential proxy for any dependence on $\mathrm{U}$. Second, our

538 database likely contains multiple ripple sizes at similar hydrodynamic conditions,

539 resulting in the lack of a clear velocity threshold. Third, we focus on developing a

540 continuous predictor so do not include any logical statements that can accommodate a

541 threshold.

542 Our results show that ripple height is more difficult to predict than ripple length

543 (e.g., Yan et al 2008; Williams et al 2004). As mentioned previously, this is likely a

544 consequence of ripple crests being subject to higher flow velocities and suspension

545 processes. Yet successful height and steepness determination is important for the

546 prediction of sediment transport, in particular the reference concentration (e.g., Green and

547 Black 1999) and sediment diffusivity (e.g., Nielsen 1992, Thorne et al., 2009). Only 2

548 equations are needed to predict height, length and steepness of ripples, but error in the

549 two chosen predicted parameters cascades to the third. The basic linear convolution of

550 predicted $\lambda(9)$ and predicted $\eta$ (11) demonstrate this cascading error: the resultant

551 steepness predictor (12) produces results that are solely dependent on grain size. We

552 instead offer 3 separate equations in the hope that workers will decide which 2 predictors

553 are most valuable for a specific research question. Notably, the GP algorithm did have

554 predicted $\lambda$ and predicted $\eta$ available as equation building blocks when determining

555 ripple steepness but the term ' $\eta / \lambda$ ' did not appear in any candidate solutions (Table 4).

556 Generating 3 separate predictors that are not self-consistent leads to geometric

557 inconsistencies, but results in better prediction for work that requires accurate prediction

558 of height and steepness but does not rely on ripple length measurements.

559 The hydrodynamic and sedimentological limit of the current prediction scheme is 
560 represented by the 4-dimensional shape that outlines the point cloud in Figure 3 (Table 1

561 contains more information regarding the range of the dataset). We excluded conditions

562 where ripples are not present either as a result of sheet flow conditions (upper plane bed)

563 or because of insufficient mobility (lower plane bed). Uncertainty in the onset of upper

564 plane bed exists because of the lack of data at a range of $\mathrm{D}_{50}$ in field-scale conditions

565 (e.g., Li and Amos, 1999; Trembanis et al., 2004; You and Yin, 2006). Additionally, field

566 work suggests that upper plane bed conditions may not be flat, but instead typified by

567 dynamic features that may be similar if not identical to long wave ripples (Green and

568 Black, 1999; Green et al., 2004; Trembanis et al., 2004). As a result of the ambiguity in

569 bed state under 'upper plane bed' conditions we did not compare our predictor to the

570 version developed by Camenen (2009), which explicitly includes a sheet flow threshold

571 (where ripples are destroyed). Furthermore we do not compare the GP derived predictors

572 with those developed by Williams et al., (2005), who developed separate predictors for

573 short wavelength and long wavelength ripples. We intentionally did not divide our

574 dataset (and develop separate predictors) in an attempt to construct a practical prediction

575 scheme that spans a wide range of conditions. Since this study aims to produce a

576 continuous predictor of wave ripple geometry, the use of discontinuous functions (logical

577 statements: e.g. 'if-then-else') has not been explored quantitatively in this contribution.

578 This study does not tackle the issue of time dependent adjustment of bedforms in

579 unsteady flow (Austin et al., 2007, Soulsby et al., 2012, Traykovski 2007; Davis et al.,

580 2004; Doucette and O’Donoghue, 2006; Hay 2008), the importance of initial conditions

581 on final ripple configuration (Traykovski et al., 1999; Hansen et al., 2001), or the explicit

582 incorporation of emergent ripple parameters (e.g., defect density; Skarke and Trembanis, 
583 2011).

584

585

5.2 Open Research Questions: Wave Ripples

586 Data integration campaigns can highlight gaps in knowledge. The collection of new

587 ripple datasets will be able to be used as either independent tests of the predictors

588 developed in this study (if the setting corresponds to an area in figure 3 that is dense with

589 points) or as new data to train the GP algorithm (if the data correspond to unexplored or

590 sparse area in figure 3; Bowden et al., 2012). Additional datasets of wave ripple geometry

591 that include more input parameters (e.g. measures of grain sorting, wave irregularity,

592 initial conditions, time dependence) are needed if prediction accuracy is to increase.

593 Furthermore datasets that encompass coarse grained environments (coarse sand and

594 gravel) and datasets in energetic conditions are still needed. Though coarse grained

595 conditions reflect a smaller fraction of the seabed than fine grained settings, coarse

596 grained environments are likely important nursery habitat for fish (Hallenbeck et al.,

597 2012). Collection of this data will not only help in the determination of ripple

598 configuration under these specific forcing conditions, but linking these environments

599 with the present data will allow for the development of a better predictor by defining the

600 shape of the prediction surface over a greater extent in phase space.

601 Conditions with waves and currents (e.g., Lacy et al., 2007; Khelifa and Ouellet,

602 2000; Arnott and Southard, 1990) are excluded from this analysis. The collection and

603 integration of data with waves and currents may lead to a more universal bedform

604 predictor in the future but more data on ripple geometry under wave and current forcing

605 is needed for machine learning techniques to be applied successfully. The dataset in this 
606 study uses median grain size as the sole sedimentological metric for predicting ripple

607 geometry. Yet many field settings may not be accurately described by a sharp peaked

608 unimodal distribution of grain sizes, and therefore prediction of ripples using $\mathrm{D}_{50}$ may

609 lead to significant error. Foti and Blondeaux (1995) showed that the addition of coarse

610 sediment can act as stabilizing feature, enhancing ripple length. It is possible that graded

611 sediment will not conform to the predictive tools outlined above. But what is the effective

$612 \mathrm{D}_{50}$ in graded sediment when predicting ripples? Furthermore variations in grain shape

613 and bed porosity may also impact the geometry of ripples. More research is needed into

614 the role of mixed grains in determining equilibrium wave ripple geometry (e.g. Calantoni

615 et al., 2013).

616 More studies are needed to better constrain thresholds between short 'anorbital'

617 ripples and large 'orbital' scale ripples (Pedocchi and García, 2009a, 2009b; Cummings

618 et al., 2009; Maier and Hay, 2009). Experimental work has thus far shown that there

619 exists no intermediate scale between these two configurations (Dumas et al., 2005;

620 Cummings et al., 2009). The determination of when large ripples appear and when

621 superimposed short ripples disappear will allow the pruning of the database in regions

622 where overlapping ripple scales occur. The decision of which ripple scale to eliminate

623 when both exist is a function of the research question being studied.

624

\section{5.3 Open Research Questions: Data Driven Prediction}

626 In this contribution we demonstrate a selection technique whereby very few data

627 are used to train the GP algorithm and most data is used as validation and independent

628 testing. The training data was selected solely from variables representing the forcing 
629 conditions. As a result the training data is not representative of the entire population of

630 ripple configurations as data points that are neighbors in 'forcing space' do not necessarily

631 have similar ripple geometries. The selected training data is therefore only related to the

632 range of forcing present in the dataset, not the range of ripple geometry. Therefore we

633 believe that our sampling strategy does not bias the testing of the predictors (which relies

634 on ripple geometry) using the reserved, unselected testing data.

635 We define the testing dataset as 'independent' because it was not shown to the GP

636 algorithm. Additionally we performed experiments by removing several individual

637 datasets from the composite dataset. The removed data serves as testing data that is not

638 shown to the selection routine, not shown to the GP algorithm, and additionally not

639 related to data shown to the selection routine/GP (this is another definition of

640 'independent'). The resultant predictors (not shown) were quantitatively similar to those

641 presented in this contribution and similarly performed better than the Pedocchi and

642 García (2009a) and Soulsby and Whitehouse (2005) predictors using only the smaller

643 sample of removed datasets as testing. Is it enough for testing data to be unseen by the

644 ML algorithm, or do entire datasets need to be reserved whole as testing data? More

645 investigation will resolve this issue.

646 Even though we were able to obtain good results using few centroids, we are

647 unaware of a technique for quantitatively determining the optimal number of centroids to

648 capture the variability in the data set while leaving the maximum amount of data for use

649 as validation/testing. Furthermore many selection and clustering routines are available,

650 and it is unclear which routine is optimal for a given dataset. It is likely that some of the

651 answers to these questions lie in statistical science and computer science literature that 
652 has not fully percolated into the Earth Sciences.

653 Our observations with the GP software show too few centroids tend to underfit the

654 data because the GP has too little training data to develop applicable solutions. With few

655 training data solutions tend to be linear and have low RMSE when compared tot the

656 validation and testing datasets. Training datasets that are larger than used in this study (>

65740 centroids) tend to produce more large (complexity $>30$ ) nonlinear solutions. In

658 addition, the solutions at complexity less than 30 are similar (if not identical) to the

659 solutions in this study (using smaller training datasets). The invariance of solutions gives

660 qualitative justification to the number of centroids used in this study, but we do not offer

661 a quantitative technique for determining the minimum number of training data needed to

662 capture dataset variability. Furthermore how is this number linked to the quantity and

663 quality of the data in the training dataset? Lastly, the stability and final criterion for

664 selecting a single predictor is subjective and can likely be improved or quantitatively

665 justified by implementing more sophisticated accounting techniques based on

666 information such as the Aikake Information Criterion (AIC) or the Bayesian Information

667 Criterion (BIC).

668

669 6. Conclusion

670 We develop equilibrium predictors of oscillatory ripple geometry using genetic

671 programming. Ripple length is a weak nonlinear function grain size and bottom orbital

672 excursion. Ripple height and steepness are nonlinear functions of grain size and predicted

673 ripple length (i.e. grain size and bottom orbital excursion). Furthermore these new

674 predictor encompass a wide range of hydrodynamic and sedimentological conditions not 
675 previously included in published prediction schemes. However, the proposed method is

676 not suitable for practical applications with significant currents present, nor under

677 conditions that would either be below the threshold of motion or above the threshold of

678 ripple wash-out. Such conditions should be identified separately by existing methods

679 (Nielsen, 1992; Lacy et al., 2007; Camenen, 2009; Soulsby et al., 2012)

680 This contribution further demonstrates the viability of developing empirical

681 predictors through ML techniques. As previously mentioned by Oehler et al., (2012), ML

682 algorithms could be integrated into future morphodynamics models (model-data fusion

683 and the development of a 'hybrid' model; Krasnapolsky and Fox-Rabinovitz, 2006),

684 replacing functions with large uncertainty.

685 The data integration campaign (which preceded the implementation of the GP

686 algorithm) had the side benefit of highlighting the current state of our knowledge on

687 ripple geometry, potentially motivating targeted data collection campaigns. Newly

688 collected data can be fed back into the GP software to develop revised predictors.

690 Acknowledgments: We thank Paula Camus for sharing her MDA routine, Malcolm 691 Green for insightful comments at the beginning of this study, and three anonymous 692 reviewers for critical feedback. EBG thanks 'IH Cantabria' for funding during his stay, 693 where part of this work was completed. G.C. acknowledges funding from the "Cantabria 694 Campus Internacional, Augusto Gonzalez Linares Program“.

695

696

697

698

699

700

701

702

703

704

705

\section{References}

Ardhuin, F., Drake, T.G., Herbers, T.H.C., 2002. Observations of wave-generated vortex ripples on the North Carolina continental shelf. Journal of Geophysical Research 107(C10), 3143, doi:10.1029/2001JC000986.

Arnott, R.W., Southard, J.B., 1990. Exploratory flow-duct experiments on combinedflow bed configurations, and some implications for interpreting storm-event stratification. Journal of Sedimentary Research 60(2), 211-219.

Austin, M.J., Masselink, G., O'Hare, T.J., Russell, P.E., 2007. Relaxation time effects of wave ripples on tidal beaches. Geophysical Research Letters 34, L16606, doi:10.1029/2007GL030696.

(C) 2017. This manuscript version is made available under the CC-BY-NC-ND 4.0 license

http://creativecommons.org/licenses/by-nc-nd/4.0/ 
706

Baptist, M.J., Babovic, V., Uthurburu, J.R., Keijzer, M., Uittenbogaard, R.E., Mynett, A., Verwey, A., 2007. On inducing equations for vegetation resistance. Journal of Hydraulic Research 45, 435-450.

Becker, J.M., Firing, Y.L., Aucan, J., Holman, R., Merrifield, M., Pawlak, G., 2007. Video-based observations of nearshore sand ripples and ripple migration. Journal of Geophysical Research 112, C01007, doi:10.1029/2005JC003451. .

Blondeaux, P., 2001. Mechanics of coastal forms. Annual Review of Fluid Mechanics 33, 339-370.

Bolaños, R., Thorne, P.D., Wolf, J., 2012. Comparison of measurements and models of bed stress, bedforms and suspended sediments under combined currents and waves. Coastal Engineering 62, 19-30.

Bowden, G.J., Maier, H.R., Dandy, G.C., 2002. Optimal division of data for neural network models in water resources applications. Water Resources Research 38(2), doi:10.1029/2001WR000266.

Bowden, G.J., Maier, H.R., Dandy, G.C., 2012. Real-time deployment of artificial neural network forecasting models: Understanding the range of applicability. Water Resources Research 48 W10549, doi:10.1029/2012WR011984.

Boyd, R., Forbes, D.L., Heffler, D.E., 1988. Time-sequence observations of wave-formed sand ripples on an ocean shoreface. Sedimentology 35, 449-464.

Calantoni, J., Landry, B.J. and Penko, A.M., 2013. Laboratory observations of sand ripple evolution using bimodal grain size distributions under asymmetric oscillatory flows In: Conley, D.C., Masselink, G., Russell, P.E. and O'Hare, T.J. (eds.), Proceedings $12^{\text {th }}$ International Coastal Symposium (Plymouth, England), Journal of Coastal Research, Special Issue No. 65, pp. 1497-1502, ISSN 07490208 .

Camenen, B., 2009. Estimation of the wave-related ripple characteristics and induced bed shear stress. Estuarine, Coastal and Shelf Science 84, 553-564.

Camus, P., Mendez, F.J., Medina, R., Cofiño, A.S., 2011. Analysis of clustering and selection algorithms for the study of multivariate wave climate. Coastal Engineering 58, 453-462.

Chang, Y.S., Hanes, D.M., 2004. Suspended sediment and hydrodynamics above mildly sloped long wave ripples. Journal of Geophysical Research 109, C07022, doi:10.1029/2003JC001900.

Charru, F., Hinch, E.J., 2006. Ripple formation on a particle bed sheared by a viscous liquid. Part 2. Oscillating flow. Journal of Fluid Mechanics 550, 123-137.

Chou, Y.J., Fringer, O.B., 2010. A model for the simulation of coupled flow-bed form evolution in turbulent flows. Journal of Geophysical Research 115, C10041, doi:10.1029/2010JC006103.

Clifton, H.E., 1976. Wave-formed sedimentary structures: A conceptual model, in Beach and Nearshore Sedimentation, Spec. Publ. 24, edited by R. A. Davis Jr. and R. L. Ethington, pp. 126-148, Soc. for Sediment. Geol., Tulsa, Okla.

Coulibaly, P., 2004. Downscaling daily extreme temperatures with genetic programming. Geophysical Research Letters 31, L16203, doi:10.1029/2004GL020075.

Cummings, D.I., Dumas, S., Dalrymple, R.W., 2009. Fine-grained versus coarse-grained wave ripples generated experimentally under large-scale oscillatory flow. Journal of Sedimentary Research 79, 83-93.

(C) 2017. This manuscript version is made available under the CC-BY-NC-ND 4.0 license http://creativecommons.org/licenses/by-nc-nd/4.0/ 
752

753

754

755

756

757

758

759

760

761

762

763

764

765

766

767

768

769

770

771

772

773

774

775

776

777

778

779

780

781

782

783

784

785

786

787

788

789

790

791

792

793

794

795

796

797

Davis, J.P., Walker, D.J., Townsend, M., Young, I.R., 2004. Wave-formed sediment ripples: Transient analysis of ripple spectral development. Journal of Geophysical Research 109, C07020, doi:10.1029/2004JC002307 .

Delgado Blanco, M., Bell, P., Montaliu, J., 2004. A new look to the applicability of classical models for ripple prediction. In: Proc. 29th Int. Conf. Coastal Eng. ASCE, Lisbon, Portugal, pp. 1909-1921.

Doucette, J.S., 2000. The distribution of nearshore bedforms and effects on sand suspension on low-energy, micro-tidal beaches in Southwestern Australia. Marine Geology 165, 41-61.

Doucette, J.S., 2002. Geometry and grain-size sorting of ripples on low-energy sandy beaches: field observations and model predictions. Sedimentology 49, 483-503.

Doucette, J.S., O'Donoghue, T., 2006. Response of sand ripples to change in oscillatory flow. Sedimentology 53, 581-596.

Dumas, S., Arnott, R.W.C., Southard, J.B., 2005. Experiments on oscillatory-flow and combined-flow bed forms: implications for interpreting parts of the shallowmarine sedimentary record. Journal of Sedimentary Research 75, 501-513.

Faraci, C., Foti, E., 2001. Evolution of small scale regular patterns generated by waves propagating over a sandy bottom. Physics of Fluids 13, 1624-1634.

Faraci, C., Foti, E., 2002. Geometry, migration and evolution of small-scale bedforms generated by regular and irregular waves. Coastal Engineering 47, 35-52.

Forbes, D.L., Boyd, R., 1987. Gravel ripples on the inner Scotian Shelf. Journal of Sedimentary Research 57(1) 46-54.

Foti, E., Blondeaux, P., 1995. Sea ripple formation: the heterogeneous sediment case. Coastal Engineering 25, 237-253.

Gonçalves, I., Silva, S., Melo, J., Carreiras, J., 2012. Random sampling technique for overfitting control in genetic programming. Genetic Programming. In: Moraglio, A., Silva, S., Krawiec, K., Machado, P., Cotta, C. (eds.) EuroGP 2012. LNCS, vol. 7244, pp. 218-229. Springer, Heidelberg .

Grant, W.D., Madsen, O.S., 1982. Movable bed roughness in unsteady oscillatory flow. Journal of Geophysical Research 87, 469-481.

Grasmeijer, B.T., Kleinhans, M.G., 2004. Observed and predicted bed forms and their effect on suspended sand concentrations. Coastal Engineering 51, 351-371.

Green, M.O., Black, K.P., 1999. Suspended-sediment reference concentration under waves: field observations and critical analysis of two predictive models. Coastal Engineering 38, 115-141.

Green, M.O., Vincent, C.E., Trembanis, A.C., 2004. Suspension of coarse and fine sand on a wave-dominated shoreface, with implications for the development of rippled scour depressions. Continental Shelf Research 24, 317-335.

Hallenbeck, T.R., Kvitek, R.G., Lindholm, J., 2012. Rippled scour depressions add ecologically significant heterogeneity to soft-bottom habitats on the continental shelf. Marine Ecology Progress Series 468: 119-133.

Hanes, D.M., Alymov, V., Chang, Y.S., Jette, C., 2001. Wave-formed sand ripples at Duck, North Carolina. J. Geophys. Res 106, 22575-22592.

Hansen, J.L., v. Hecke, M., Ellegaard, C., Andersen, K.H., Bohr, T., Haaning, A., Sams, T., 2001. Stability balloon for two-dimensional vortex ripple patterns. Physical Review Letters 87.

(C) 2017. This manuscript version is made available under the CC-BY-NC-ND 4.0 license http://creativecommons.org/licenses/by-nc-nd/4.0/ 
798

799

800

801

802

803

804

805

806

807

808

809

810

811

812

813

814

815

816

817

818

819

820

821

822

823

824

825

826

827

828

829

830

831

832

833

834

835

836

837

838

839

840

841

842

843

Hay, A.E., 2008. Near-bed turbulence and relict waveformed sand ripples: Observations from the inner shelf. Journal of Geophysical Research 113, C04040, doi:10.1029/2006JC004013.

Hume, T.M., Green, M.O., Oldman, J.W., 1999. What happens at the seabed off a headland during a tropical cyclone, Coastal Sediments '99, Am. Soc. of Civ. Eng., Reston, Va. pp. 1836-1851.

Inman, D.L., 1957. Wave-generated ripples in nearshore sands, Tech. Memo. 100, Dep. of the U. S. Army Corps of Eng., Washington, D. C.

Kambekar, A.R., Deo, M.C., 2012. Wave prediction using genetic programming and model trees. Journal of Coastal Research 28, 43-50.

Kennedy, J. F., Falcon, M., 1965. Wave generated sediment ripples, Rep. 86, Hydrodyn. Lab., Mass. Inst. of Technol., Cambridge.

Khelifa, A., Ouellet, Y., 2000. Prediction of sand ripple geometry under waves and currents. Journal of waterway, port, coastal, and ocean engineering 126, 14-22.

Koza, J.R., 1992. Genetic Programming, On the Programming of Computers by Means of Natural Selection. MIT Press, Cambridge, MA, USA.

Krasnopolsky, V.M., Fox-Rabinovitz, M.S., 2006. A new synergetic paradigm in environmental numerical modeling: Hybrid models combining deterministic and machine learning components. Ecological Modelling 191, 5-18.

Kushchu, I., 2002. An evaluation of evolutionary generalisation in genetic programming. Artificial Intelligence Review 18, 3-14.

Lacy, J.R., Rubin, D.M., Ikeda, H., Mokudai, K., Hanes, D.M., 2007. Bed forms created by simulated waves and currents in a large flume. Journal of Geophysical Research 112, C10018, doi:10.1029/2006JC003942.

Leckie, D., 1988. Wave-formed, coarse-grained ripples and their relationship to hummocky cross-stratification. Journal of Sedimentary Research 58.

Li, M.Z., Amos, C.L., 1999. Sheet flow and large wave ripples under combined waves and currents: field observations, model predictions and effects on boundary layer dynamics. Continental Shelf Research 19, 637-663.

Maier, I., Hay, A.E., 2009. Occurrence and orientation of anorbital ripples in near-shore sands. Journal of Geophysical Research 114, F04022, doi:10.1029/2008JF001126.

Marieu, V., Bonneton, P., Foster, D.L., Ardhuin, F., 2008. Modeling of vortex ripple morphodynamics. Journal of Geophysical Research 113, C09007.

Masselink, G., Austin, M.J., O'Hare, T.J., Russell, P.E., 2007. Geometry and dynamics of wave ripples in the nearshore zone of a coarse sandy beach. Journal of Geophysical Research 112, C10022, doi:10.1029/2006JC003839.

May, R.J., Maier, H.R., Dandy, G.C., 2010. Data splitting for artificial neural networks using SOM-based stratified sampling. Neural Networks 23, 283-294.

Miller, M.C., Komar, P.D., 1980a. A field investigation of the relationship between oscillation ripple spacing and the near-bottom water orbital motions. Journal of Sedimentary Research 50(1) 173-182.

Miller, M.C., Komar, P.D., 1980b. Oscillation sand ripples generated by laboratory apparatus. Journal of Sedimentary Research 50(1) 183-191.

Mogridge, G.R., 1972. Wave generated bed forms, Ph.D. thesis, Queens Univ., Kingston, Ont., Canada.

Mogridge, G.R., Davies, M.H., Willis, D.H., 1994. Geometry prediction for wave-

(C) 2017. This manuscript version is made available under the CC-BY-NC-ND 4.0 license

http://creativecommons.org/licenses/by-nc-nd/4.0/ 
844

generated bedforms. Coastal Engineering 22, 255-286.

Nielsen, P., 1981. Dynamics and geometry of wave-generated ripples. Journal of Geophysical Research 86, 6467-6472.

Nielsen, P., 1992. Coastal bottom boundary layers and sediment transport. World Scientific Publishing Company Incorporated.

O'Donoghue, T., Clubb, G.S., 2001. Sand ripples generated by regular oscillatory flow. Coastal Engineering 44, 101-115.

O'Donoghue, T., Doucette, J.S., Van der Werf, J.J., Ribberink, J.S., 2006. The dimensions of sand ripples in full-scale oscillatory flows. Coastal Engineering 53, 997-1012.

O’Neill, M., Vanneschi, L., Gustafson, S., Banzhaf, W., 2010. Open issues in genetic programming. Genetic Programming and Evolvable Machines 11, 339-363.

Oehler, F., Coco, G., Green, M.O., Bryan, K.R., 2012. A data-driven approach to predict suspended-sediment reference concentration under non-breaking waves. Continental Shelf Research 46, 96-106.

Pape, L., Kuriyama, Y., Ruessink, B.G., 2010. Models and scales for cross-shore sandbar migration. Journal of Geophysical Research 115, F03043.

Pedocchi, F., García, M.H., 2009a. Ripple morphology under oscillatory flow: 1. Prediction. Journal of Geophysical Research 114, C12014, doi:10.1029/2009JC005354.

Pedocchi, F., García, M.H., 2009b. Ripple morphology under oscillatory flow: 2. Experiments. Journal of Geophysical Research 114, C12015, doi:10.1029/2009JC005356.

Poli, R., Langdon, W.B., McPhee, N.F., 2008. A field guide to genetic programming. Lulu Enterprises Uk Limited.

Ribberink, J.S., Al-Salem, A.A., 1994. Sediment transport in oscillatory boundary layers in cases of rippled beds and sheet flow. Journal of Geophysical Research 99, 12707-12712.

Schmidt, M., Lipson, H., 2009. Distilling free-form natural laws from experimental data. Science 324, 81-85.

Schmidt, M., Lipson, H., 2013. Eureqa (Version 0.98 beta) [Software]. Available from http://www.eureqa.com/

Silva, S., Tseng, Y.T., 2008. Classification of Seafloor Habitats Using Genetic Programming. In: Giacobini, M., Brabazon, A., Cagnoni, S., Di Caro, G.A., Drechsler, R., Ek' art, A., Esparcia-Alc' azar, A.I., Farooq, M., Fink, A., McCormack, J., O’Neill, M., Romero, J., Rothlauf, F., Squillero, G., Uyar, A.S ,, Yang, S. (eds.) EvoWorkshops 2008. LNCS, vol. 4974, pp. 315-324. Springer, Heidelberg.

Skarke, A., Trembanis, A.C., 2011. Parameterization of bedform morphology and defect density with fingerprint analysis techniques. Continental Shelf Research 31, 16881700.

Sleath, J.F.A., 1982. The suspension of sand by waves. Journal of Hydraulic Research 20, 439-452.

Sleath, J.F.A., Wallbridge, S., 2002. Pickup from rippled beds in oscillatory flow. Journal of Waterway, Port, Coastal, and Ocean Engineering 128, 228-237.

Smith, J. J., Wiberg, P. L., 2006. Ripple Geometry in Wave-Dominated Environments 
Revisited, paper presented at Eos Trans. AGU, 87(36), Ocean Sci. Meet. Suppl., Abstract OS35D-24.

Soulsby, R.L., Whitehouse, R.J.S., 2005. Prediction of ripple properties in shelf seas. Mark 2 Predictor for time evolution. Report TR154, HR Wallingford, Wallingford, UK. http://eprints.hrwallingford.co.uk/281/

Soulsby, R.L., Whitehouse, R.J.S., Marten, K.V., 2012. Prediction of time-evolving sand ripples in shelf seas. Continental Shelf Research 38, 47-62.

Southard, J. B., Lambie, J., Federico, D., Pile, H., Weidman, C., 1990. Experiments on bed configurations in fine sands under bidirectional purely oscillatory flow, and the origin of hummocky cross-stratification. Journal of Sedimentary Petrology, 60(1), $1-17$

Styles, R., Glenn, S.M., 2002. Modeling bottom roughness in the presence of wavegenerated ripples. Journal of Geophysical Research, 107(C8), doi:10.1029/2001JC000864

Thorne, P.D., Williams, J.J., Davies, A.G., 2002. Suspended sediments under waves measured in a large-scale flume facility. Journal of Geophysical Research, 107(C8), doi:10.1029/2001JC000988

Thorne, P.D., Davies, A.G., Bell, P.S., 2009. Observations and analysis of sediment diffusivity profiles over sandy rippled beds under waves. Journal of Geophysical Research, 114, C02023, doi:10.1029/2008JC004944

Traykovski, P., 2007. Observations of wave orbital scale ripples and a nonequilibrium time-dependent model. Journal of Geophysical Research 112, C06026, doi:10.1029/2006JC003811.

Traykovski, P., Hay, A.E., Irish, J.D., Lynch, J.F., 1999. Geometry, migration, and evolution of wave orbital ripples at LEO-15. Journal of Geophysical Research 104(C1), 1505-1524, doi:10.1029/1998JC900026.

Trembanis, A.C., Wright, L.D., Friedrichs, C.T., Green, M.O., Hume, T., 2004. The effects of spatially complex inner shelf roughness on boundary layer turbulence and current and wave friction: Tairua embayment, New Zealand. Continental Shelf Research 24, 1549-1571.

van Maanen, B., Coco, G., Bryan, K.R., Ruessink, B.G., 2010. The use of artificial neural networks to analyze and predict alongshore sediment transport. Nonlinear Processes in Geophysics: 17, p. 395-404. doi:10.5194/npg-17-395-2010

Van Rijn, L.C., 1993. Principles of sediment transport in rivers, estuaries and coastal seas. Aqua publications Amsterdam.

Werner, B.T., Kocurek, G., 1999. Bedform spacing from defect dynamics. Geology 27, 727-730.

Whigham, P.A., Recknagel, F., 1999. Predictive modelling of plankton dynamics in freshwater lakes using genetic programming. In: MODSIM 1999 International Congress on Modelling and Simulation, Modeling and Simulation Society of Australia and New Zealand, 6-9 December, 1999, New Zealand, pp. 691-696.

Wiberg, P.L., Harris, C.K., 1994. Ripple geometry in wave-dominated environments. Journal of Geophysical Research 99(C1), 775-775, doi:10.1029/93JC02726.

Wikramanayake, P.N., Madsen, O.S., 1991. Calculation of movable bed friction factors. Technical Report. Massachusetts Inst. of Technol., Cambridge, Massachusetts, $105 \mathrm{pp}$. 
Williams, J.J., Bell, P.S., Thorne, P.D., Trouw, K., Hardcastle, P.J., Humphery, J.D., 2000. Observed and predicted vertical suspended sediment concentration profiles and bedforms in oscillatory-only flow. Journal of Coastal Research, 698-708.

Williams, J.J., Bell, P.S., Thorne, P.D., 2005. Unifying large and small wave-generated ripples. Journal of Geophysical Research 110, C02008, doi:10.1029/2004JC002513.

Williams, J.J., Bell, P.S., Thorne, P.D., Metje, N., Coates, L.E., 2004. Measurement and prediction of wave-generated suborbital ripples. Journal of Geophysical Research 109, C02004, doi:10.1029/2003JC001882.

$\mathrm{Xu}$, J.P., 2005. Observations of plan-view sand ripple behavior and spectral wave climate on the inner shelf of San Pedro Bay, California. Continental Shelf Research 25, 373-396.

Yamaguchi, N., Sekiguchi, H., 2011. Variability of wave-induced ripple migration in wave-flume experiments and its implications for sediment transport. Coastal Engineering 58, 671-677.

Yan, B., Zhang, Q.H., Wai, O.W.H., 2008. Prediction of sand ripple geometry under waves using an artificial neural network. Computers \& Geosciences 34, 16551664.

You, Z.-J., Yin, B., 2006. A unified criterion for initiation of sediment motion and inception of sheet flow under water waves. Sedimentology 53, 1181-1190. 
959 Table 1: Data Summary; Measurement numbers reported are the ripple length

960 measurements used in our study. Measurements with both length and height are less.

\begin{tabular}{|c|c|c|c|c|c|c|}
\hline Authors & Setting & Measurements & $\mathrm{T}(\mathrm{s})$ & $\mathrm{U}(\mathrm{m} / \mathrm{s})$ & $\mathrm{d}_{0}(\mathrm{~m})$ & $\mathrm{D}_{50}(\mathrm{~m})$ \\
\hline Boyd et al. 1988 & Field & 36 & $3.8-9.8$ & $0.04-0.28$ & $0.05-0.60$ & 0.00011 \\
\hline $\begin{array}{l}\text { Cummings et al. } \\
2009\end{array}$ & $\begin{array}{l}\text { Wave } \\
\text { Racetrack }\end{array}$ & 14 & $4.4-14$ & $0.37-1.22$ & $0.95-4.50$ & $0.00012-0.0008$ \\
\hline $\begin{array}{l}\text { Delgado Blanco } \\
\text { et al. } 2004\end{array}$ & Wave Flume & 17 & 6.0 & $0.14-0.74$ & $0.27-1.42$ & 0.00035 \\
\hline Doucette 2000 & Field & 49 & $4.7-12.2$ & $0.15-0.52$ & $0.31-1.93$ & $0.00015-0.00053$ \\
\hline Doucette 2002 & Field & 25 & $2.2-12.2$ & $0.17-0.66$ & $0.31-2.22$ & $0.00035-0.00062$ \\
\hline $\begin{array}{l}\text { Doucette and } \\
\text { O'Donoghue } \\
2006\end{array}$ & Osc. Tunnel & 32 & $2.0-12.2$ & $0.29-0.63$ & $0.24-2.00$ & 0.00044 \\
\hline Dumas et al. 2005 & Osc. Tunnel & 23 & $7.9-11.0$ & $0.21-1.26$ & $0.51-4.17$ & $0.00011-0.00023$ \\
\hline $\begin{array}{l}\text { Grasmeijer and } \\
\text { Kleinhans } 2004\end{array}$ & Field & 26 & $4.0-10.5$ & $0.23-0.84$ & $0.58-2.41$ & 0.00024 \\
\hline Hanes et al. 2001 & Field & 169 & $7.1-19.7$ & $0.92-1.11$ & $0.47-5.02$ & $0.00012-0.00166$ \\
\hline Hume et al. 1999 & Field & 9 & 11.0 & $0.08-0.37$ & $0.30-1.30$ & 0.00040 \\
\hline Inman 1957 & Field & 59 & $0.5-15.0$ & $0.06-0.94$ & $0.04-2.74$ & $0.00008-0.00091$ \\
\hline $\begin{array}{l}\text { Kennedy and } \\
\text { Falcon } 1965\end{array}$ & Wave Flume & 10 & $1.1-2.0$ & $0.12-0.26$ & $0.04-0.13$ & $0.00010-0.00032$ \\
\hline $\begin{array}{l}\text { Miller and Komar } \\
\text { 1980a }\end{array}$ & Wave Flume & 4 & $3.0-8.0$ & $0.05-0.34$ & $0.14-0.54$ & 0.00017 \\
\hline $\begin{array}{l}\text { Miller and Komar } \\
\text { 1980b }\end{array}$ & Field & 26 & $6.0-18.2$ & $0.03-0.41$ & $0.07-2.14$ & $0.00017-0.00029$ \\
\hline Mogridge 1972 & $\begin{array}{l}\text { Osc. Tunnel/ } \\
\text { W. Flume }\end{array}$ & 72 & $1.0-14.0$ & $0.13-0.68$ & $0.05-1.84$ & 0.00036 \\
\hline $\begin{array}{l}\text { O'Donoghue and } \\
\text { Clubb 2001 }\end{array}$ & Osc. Tunnel & 35 & $2.0-15.0$ & $0.25-0.94$ & $0.16-2.92$ & $0.00018-0.00044$ \\
\hline $\begin{array}{l}\text { O'Donoghue et } \\
\text { al. } 2006\end{array}$ & Osc. Tunnel & 27 & $3.1-12.5$ & $0.31-0.85$ & $0.42-2.70$ & $0.00022-0.00044$ \\
\hline $\begin{array}{l}\text { Pedocchi and } \\
\text { García 2009b }\end{array}$ & Osc. Tunnel & 22 & $2.0-18.0$ & $0.20-1.00$ & $0.16-2.86$ & 0.00025 \\
\hline $\begin{array}{l}\text { Ribberink and Al- } \\
\text { Salem } 1994\end{array}$ & Osc. Tunnel & 25 & $2.0-10.0$ & $0.30-1.50$ & $0.31-3.82$ & 0.00021 \\
\hline Sleath 1982 & Osc. Tunnel & 13 & $2.9-5.1$ & $0.16-0.44$ & $0.17-0.51$ & $0.00020-0.00041$ \\
\hline $\begin{array}{l}\text { Sleath and } \\
\text { Wallbridge } 2002\end{array}$ & Osc. Tunnel & 26 & $2.8-5$ & $0.08-0.77$ & $0.12-0.80$ & $0.00020-0.00080$ \\
\hline $\begin{array}{l}\text { Southard et al. } \\
1990\end{array}$ & Osc. Tunnel & 63 & $93.1-19.3$ & $0.16-1.00$ & $0.26-3.56$ & $0.00011-0.00032$ \\
\hline Thorne et al. 2002 & Wave Flume & 14 & $4.0-6.0$ & $0.26-0.66$ & $0.41-1.05$ & 0.00033 \\
\hline $\begin{array}{l}\text { Williams et al. } \\
2000\end{array}$ & Wave Flume & 9 & $4.8-5.3$ & $0.19-0.69$ & $0.30-1.10$ & $0.00016-0.00033$ \\
\hline $\begin{array}{l}\text { Williams et al. } \\
2004\end{array}$ & Wave Flume & 65 & $4.0-6.0$ & $0.13-1.02$ & $0.25-1.96$ & $0.00016-0.00035$ \\
\hline $\mathrm{Xu} 2005$ & Field & 13 & $8.9-14.8$ & $0.11-0.16$ & $0.41-0.76$ & 0.00009 \\
\hline $\begin{array}{l}\text { Yamaguchi and } \\
\text { Sekiguchi } 2011\end{array}$ & Wave Flume & 111 & $1.3-5.0$ & $0.18-0.51$ & $0.07-0.55$ & $0.00032-0.00073$ \\
\hline
\end{tabular}

961

962

963

(C) 2017. This manuscript version is made available under the CC-BY-NC-ND 4.0 license

http://creativecommons.org/licenses/by-nc-nd/4.0/ 
965 Figure 1: A schematic phase diagram of oscillatory bedforms. O, S, and A represent

966 orbital, suborbital, and anorbital ripples respectively: smaller steep ripples that occur

967 under small/moderate hydrodynamic forcing in fine sands. Orbital, suborbital and

968 Anorbital ripples occur in sequence as hydrodynamic forcing is increased. Recent data

969 collection campaigns have focused on 1) strong hydrodynamic forcing in fine sands

970 ('hummocks' or 'long wave ripples') and 2) steep, large ripples in coarse sand. Modified

971 after Cummings et al., (2009). Question marks denote the unknown threshold for plane

972 bed in coarse grained environments, and unknown potential for coarse grained

973 environments to be sculpted into long wavelength 'hummocky' ripples. Additionally it is

974 unknown if suborbital and anorbital scale ripples exist in coarse grain settings. Lower

975 plane bed conditions are likely only applicable for laboratory studies where the bed is

976 artificially flattened (field conditions retain relict or antecedent bed geometry).

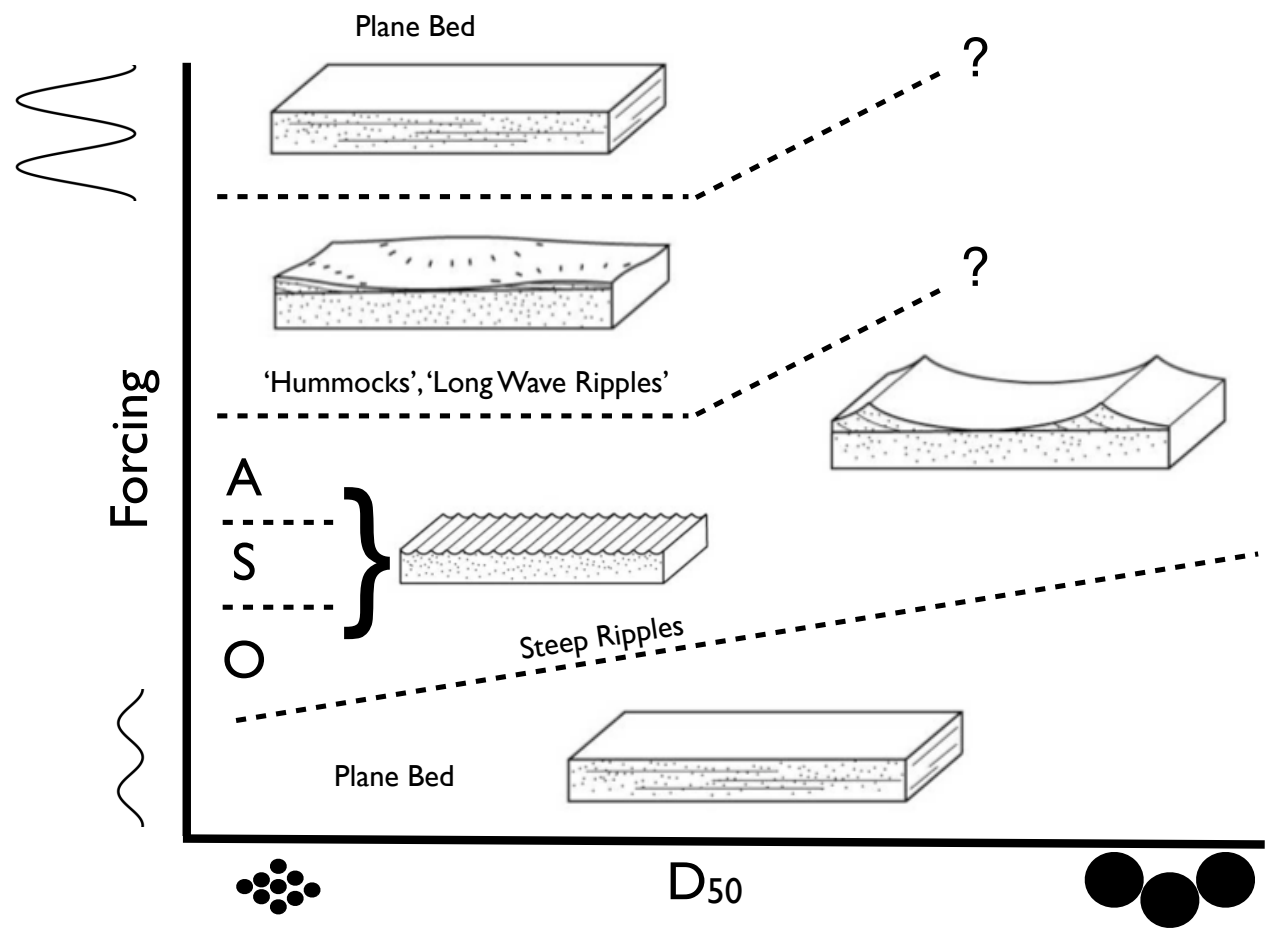

(C) 2017. This manuscript version is made available under the CC-BY-NC-ND 4.0 license http://creativecommons.org/licenses/by-nc-nd/4.0/ 
978 Figure 2: Histograms for ripple length (995 measurements), ripple height (872

979 measurements), ripple steepness (872 measurements), and for hydrodynamic and

980 sedimentological variables used in this study (includes all 995 data points). Note the

981 different $\mathrm{Y}$-axis values for each graph.
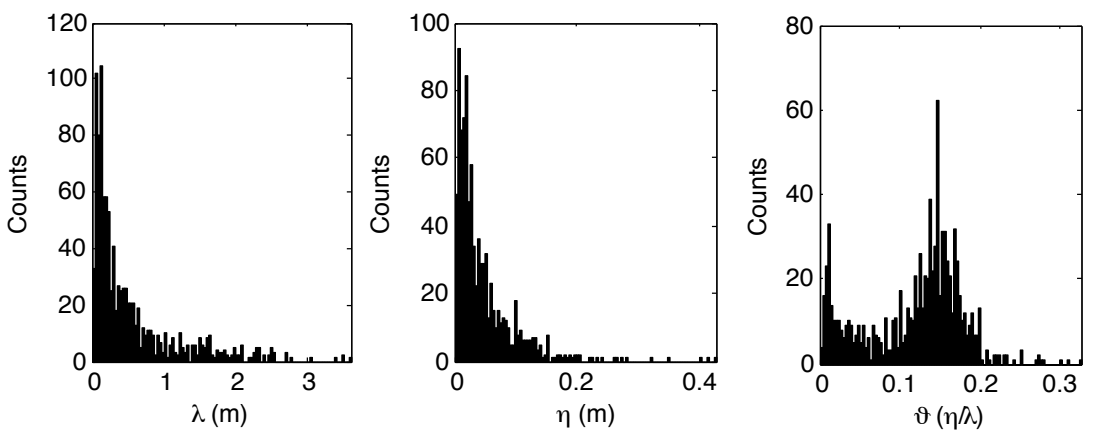

982
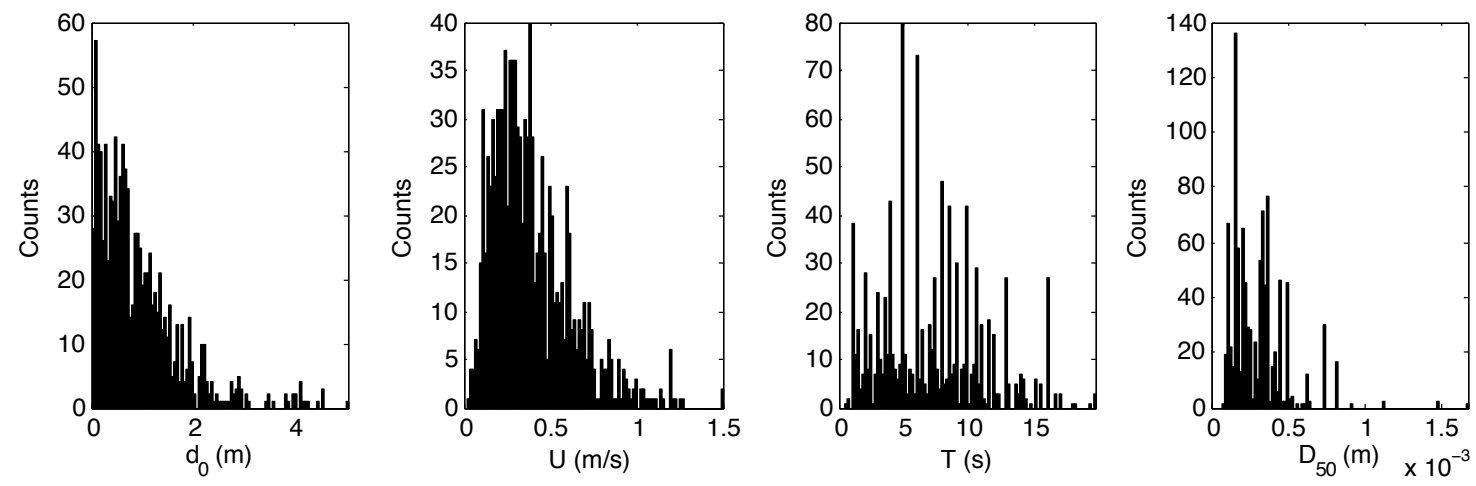

983 
984 Figure 3: Visualization of the range of forcing conditions in the ripple length dataset.

985 Each plot represents a 2 dimensional projection of the entire data set onto the set of axes

986 shown. For instance, the first panel with data projected onto the U-T plane shows no

987 information about $\mathrm{D}_{50}$ or $\mathrm{d}_{0}$. Ripple height dataset shows qualitatively similar distribution

988 and range, but with fewer data points (872 vs. 995 ).
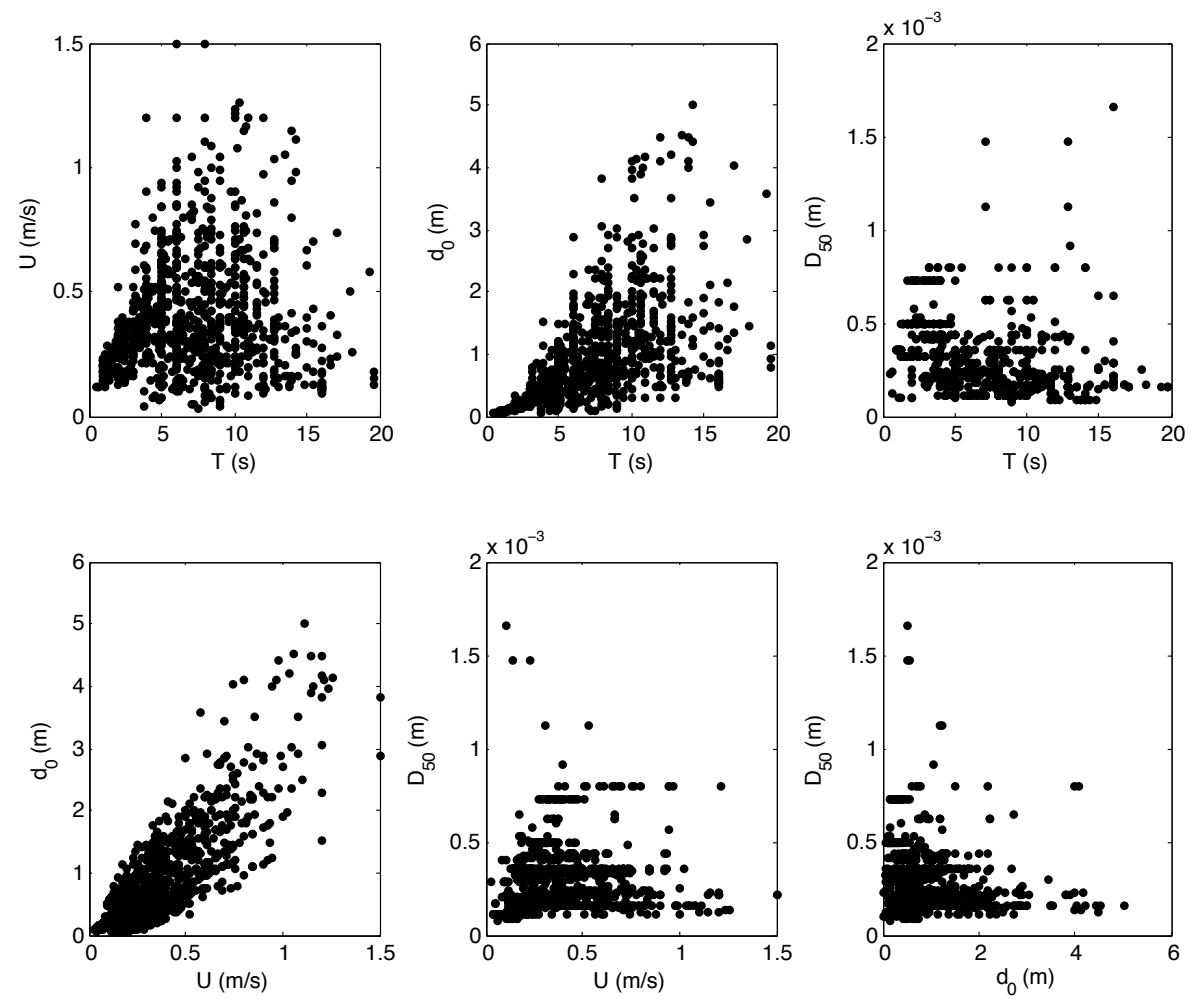

989

990 
991 Figure 4: Centroid locations in the ripple length dataset, visualized using the projections

992 shown in Figure 3. Stars denote centroid locations (training data), while points denote

993 unselected data (validation and testing). Note that centroids are distributed throughout the

994 dataset. Centroid locations for the ripple height (and steepness) dataset look qualitatively

995 similar but have more centroids (40 vs. 30) and fewer data points (832 vs. 965$)$.
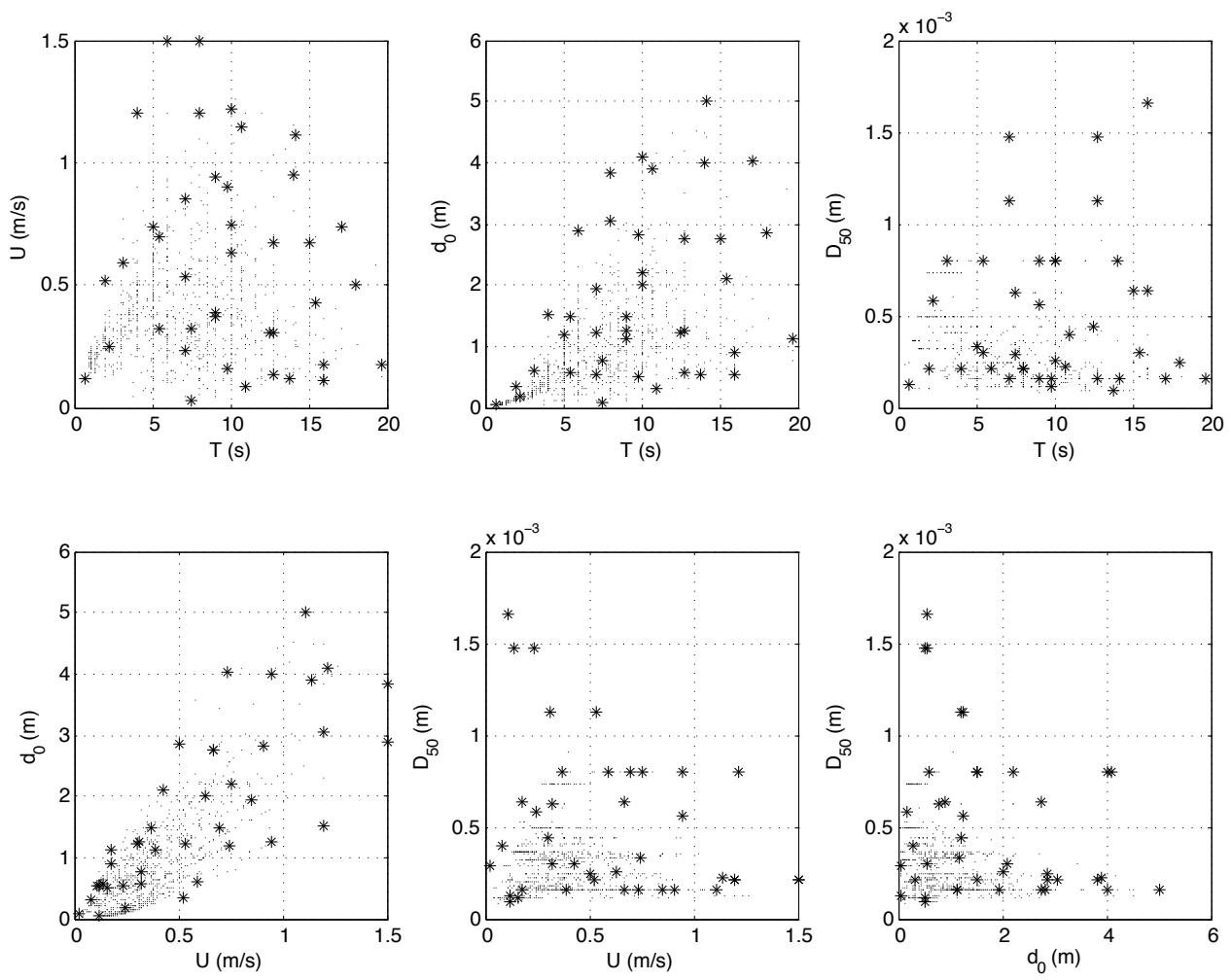

996

997

(C) 2017. This manuscript version is made available under the CC-BY-NC-ND 4.0 license http://creativecommons.org/licenses/by-nc-nd/4.0/ 
998 Figure 5: Example of the genetic programming process. Potential solutions are encoded 999 as a population of 'trees'. Here a hypothetical population of two solutions is shown. The

1000 first solution has a low MSE and therefore persists to the next iteration. The second

1001 solution has a high MSE and therefore is subject to removal, mutation, or crossover. Here

1002 is an example of 'crossover' whereby the old solution is combined with parts of other,

1003 better performing solutions to create a new potential solution in the next iteration.

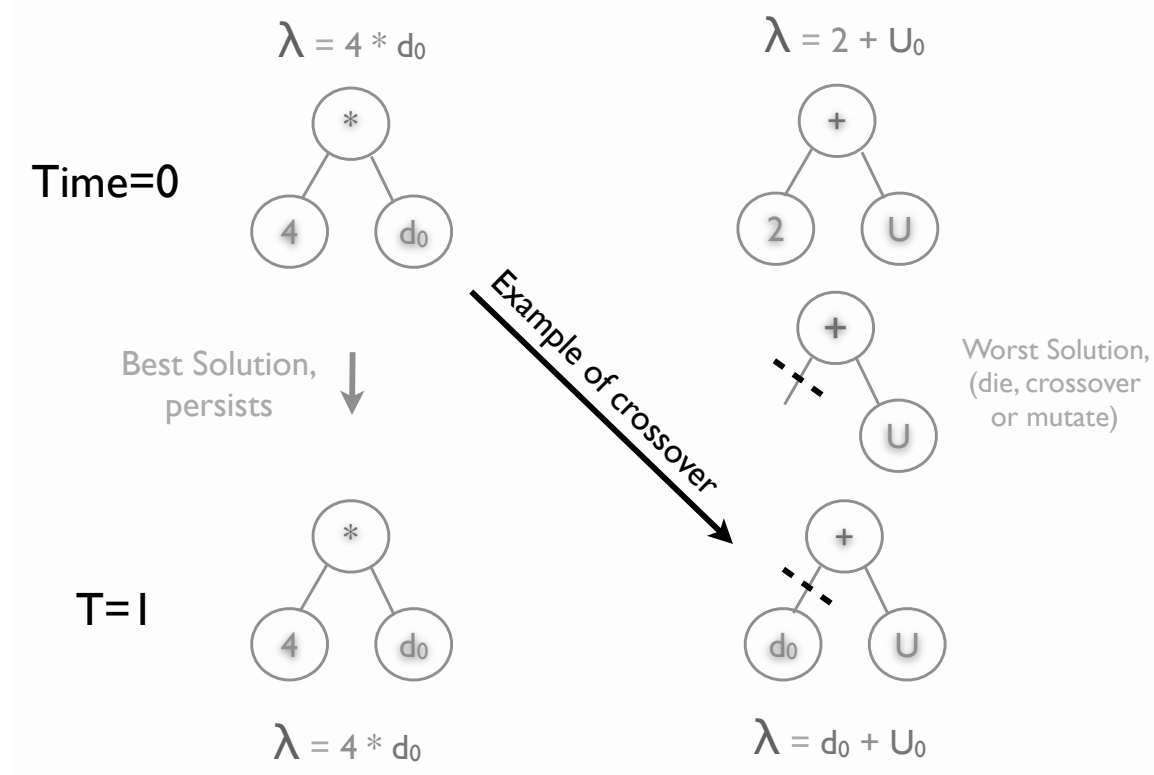

1004

1005 
1006 Figure 6: Ripple Length Pareto front; Error is expressed as mean squared error of

1007 candidate solution versus the validation data set. Complexity is a quantification of the

1008 candidate solution length (both mathematical operators and variables).

1009

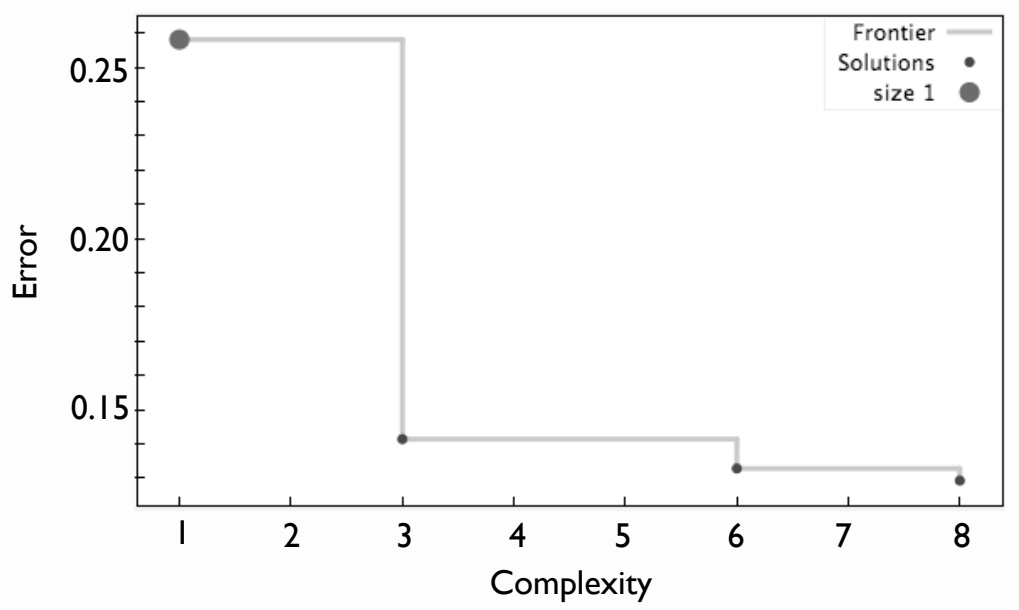

1010

1011 Table 2: Solutions for Ripple Length

\begin{tabular}{|l|l|l|}
\hline Solution & Complexity & MSE \\
\hline$\lambda=U$ & 1 & 0.258 \\
\hline$\lambda=0.607 d_{0}$ & 3 & 0.141 \\
\hline$\lambda=\frac{d_{0}}{1.39+\left(1000 D_{50}\right)}$ & 6 & 0.133 \\
\hline$\lambda=\frac{d_{0}}{1.12+2.18\left(1000 D_{50}\right)}$ & 8 & 0.129 \\
\hline
\end{tabular}

1012

1013 
1014 Figure 7: Example behavior of ripple length predictor as a function of grain size for given

1015 bottom orbital excursions (left panel) and as a function of bottom orbital excursion for

1016 given grain size (right panel).
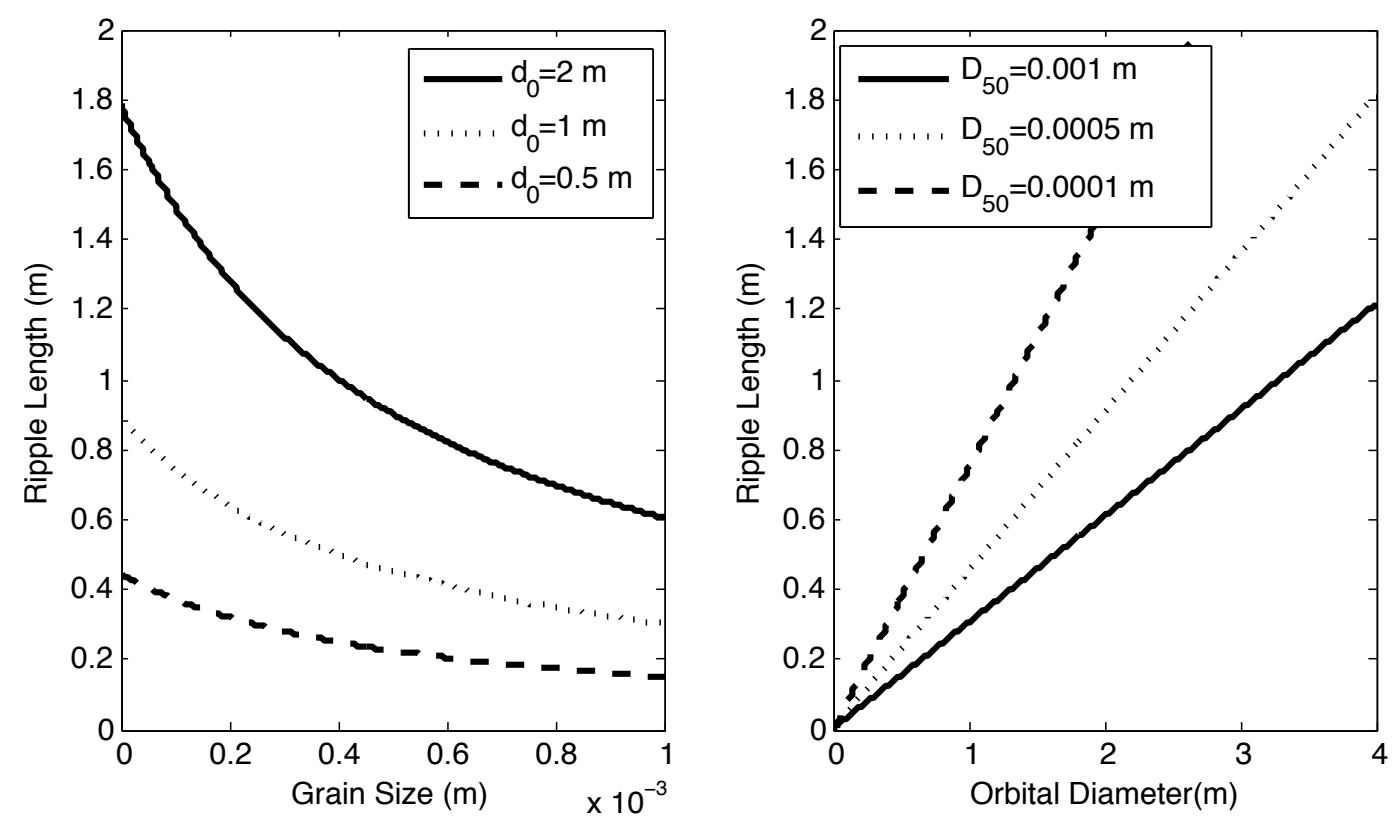

1017

1018 
1019 Figure 8: GP predictor of ripple length (8), Soulsby and Whitehouse (2005) predictor (3)

1020 and Pedocchi and García (2009a) predictor (5) evaluated using only the independent

1021 testing dataset. Top row shows the predictors in linear space, while bottom row shows

1022 log-log space.
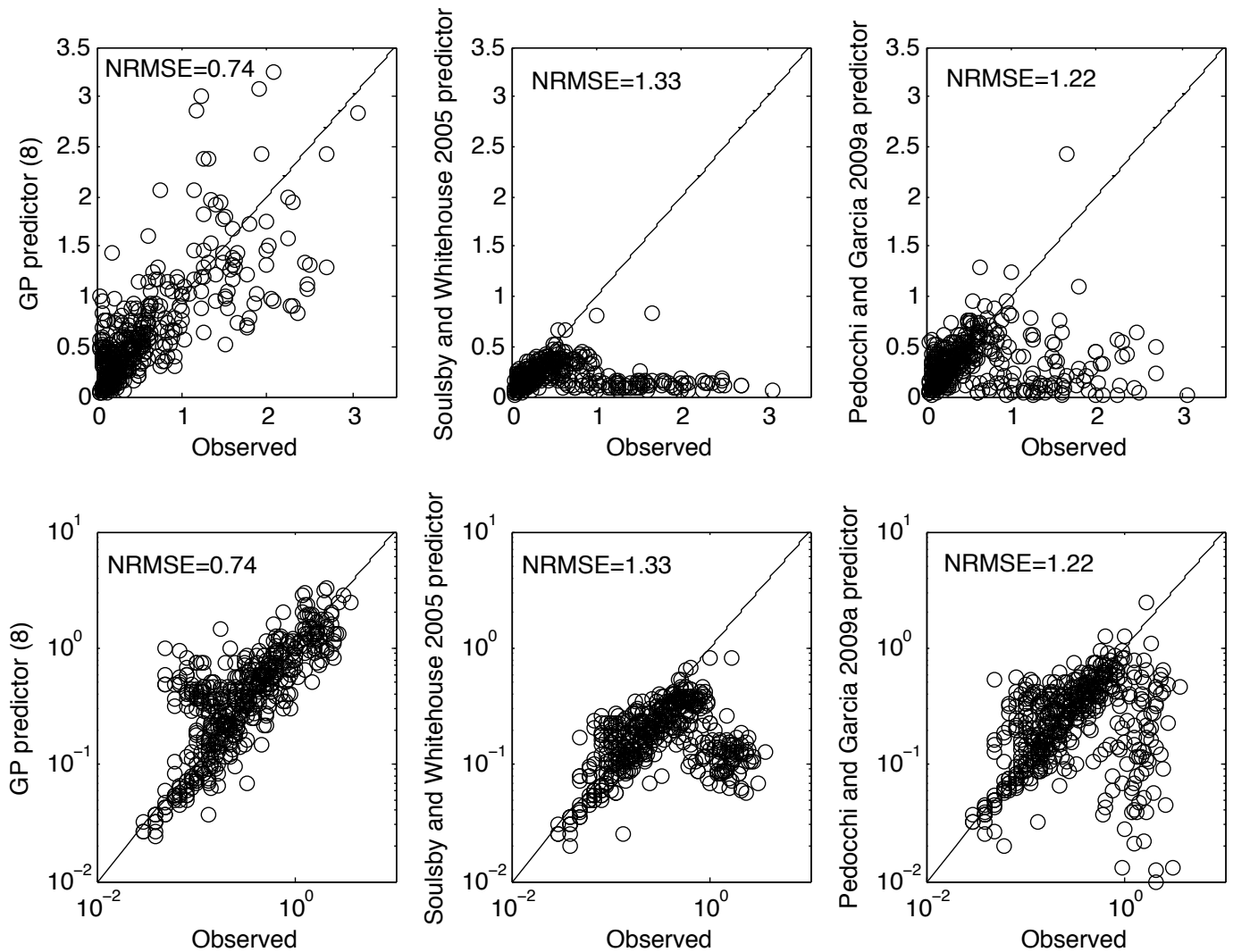

1024 
1025 Figure 9: Pareto front for ripple height; Error is mean squared error of candidate solution

1026 versus the validation data set. Complexity is a quantification of the candidate solution

1027 length (both mathematical operators and variables).

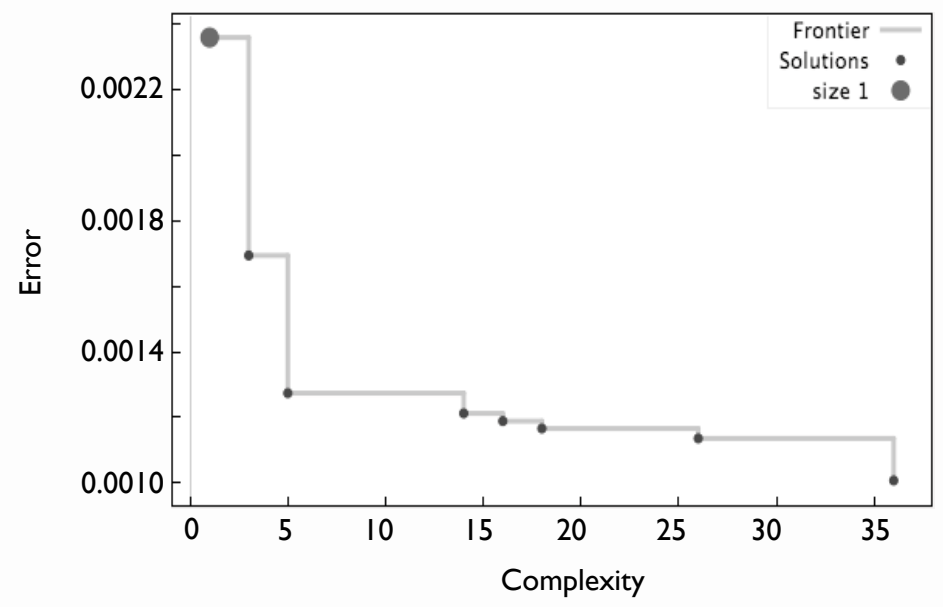

1028

1029

1030 Table 3: Solutions for Ripple Height

\begin{tabular}{|c|c|c|}
\hline Solution & $\mathrm{C}$ & MSE \\
\hline$\eta=0.435 d_{0}$ & 3 & 0.0017 \\
\hline$\eta=0.313 \lambda\left(1000 D_{50}\right)$ & 5 & 0.0013 \\
\hline$\eta=\frac{\lambda\left(1000 D_{50}\right)^{2}}{0.372+5.29\left(1000 D_{50}\right)^{2}}$ & 14 & 0.0012 \\
\hline$\eta=\frac{\lambda\left(1000 D_{50}\right)^{3}}{0.0731+5.57\left(1000 D_{50}\right)^{3}}$ & 18 & 0.0012 \\
\hline$\eta=\frac{0.0237 \lambda\left(1000 D_{50}\right)+\lambda\left(1000 D_{50}\right)^{3}-0.308 \lambda\left(1000 D_{50}\right)^{2}}{0.0332+4.46\left(1000 D_{50}\right)^{3}-0.321 D_{50}}$ & 36 & 0.0010 \\
\hline
\end{tabular}

1031

1032 
1033 Figure 10: Example behavior of Ripple height predictor as a function of grain size for 1034 given bottom orbital excursions (left panel) and as a function of bottom orbital excursion 1035 for given grain size (right panel).

1036
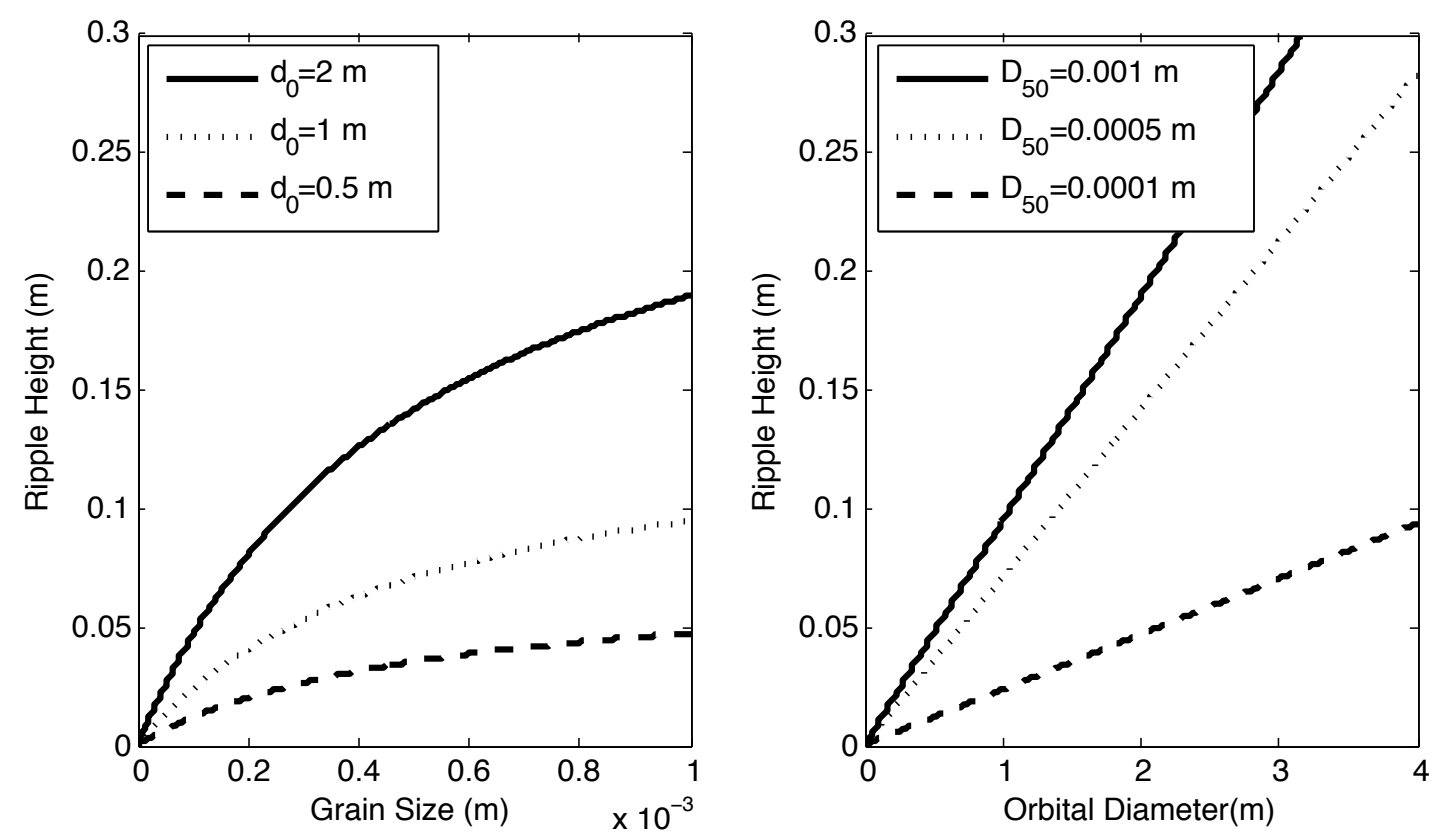

1037 
1038 Figure 11: GP predictor of ripple height (10), Soulsby and Whitehouse (2005) predictor 1039 (3) and (4) and Pedocchi and García (2009a) predictor (6) evaluated using only the 1040 independent testing dataset. Top row shows the predictors in linear space, while bottom 1041 row shows log-log space.
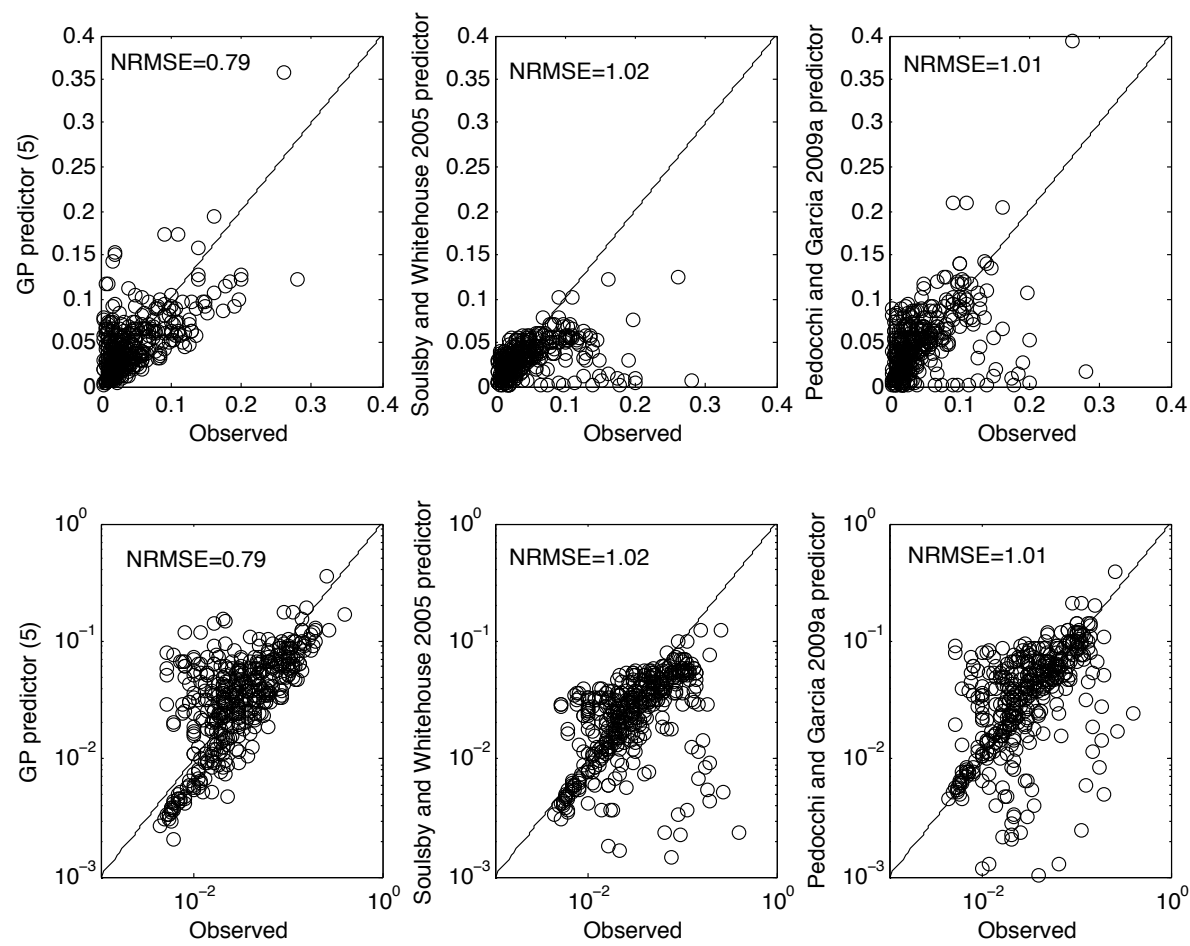

1042

1043 
1044 Figure 12: Pareto front for ripple steepness; Error is mean squared error of candidate 1045 solution versus the validation data set. Complexity is a quantification of the candidate 1046 solution length (both mathematical operators and variables).

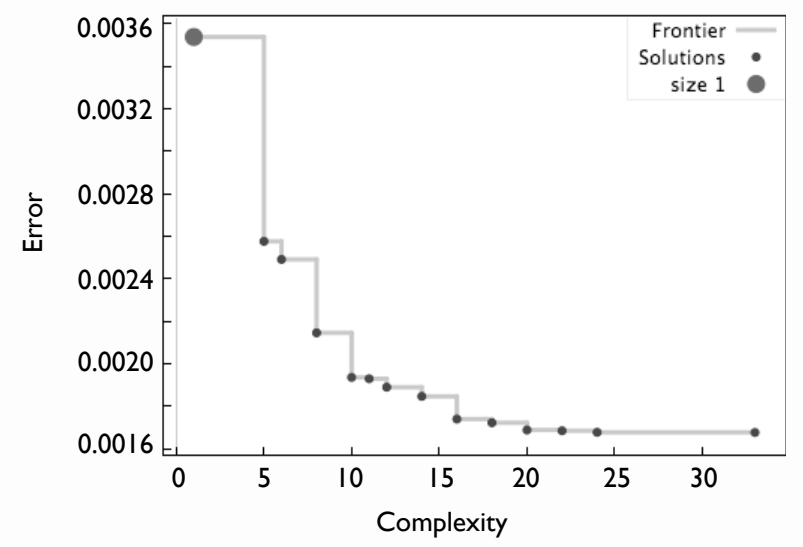

1047

1048 Table 4: Solutions for Ripple Steepness

\begin{tabular}{|l|l|l|}
\hline Solution & C & MSE \\
\hline$\vartheta=0.119$ & 1 & 0.0035 \\
\hline$\vartheta=0.154-0.0613 \lambda$ & 5 & 0.0026 \\
\hline$\vartheta=\frac{\left(1000 D_{50}\right)}{\lambda+6.23\left(1000 D_{50}\right)}$ & 8 & 0.0021 \\
\hline$\vartheta=\frac{3.42}{22+\left(\frac{\lambda}{\left(1000 D_{50}\right)}\right)^{2}}$ & 10 & 0.0019 \\
\hline$\vartheta=\frac{0.447}{2.81+\left(\lambda^{2}+\frac{-0.617 \lambda}{\left(1000 D_{50}\right)}\right)^{2}}$ & 16 & 0.0017 \\
\hline
\end{tabular}

1049

1050 
1051 Figure 13: Example behavior of Ripple Steepness predictor as a function of grain size for 1052 given bottom orbital excursions (left panel) and as a function of bottom orbital excursion 1053 for given grain size (right panel).

1054
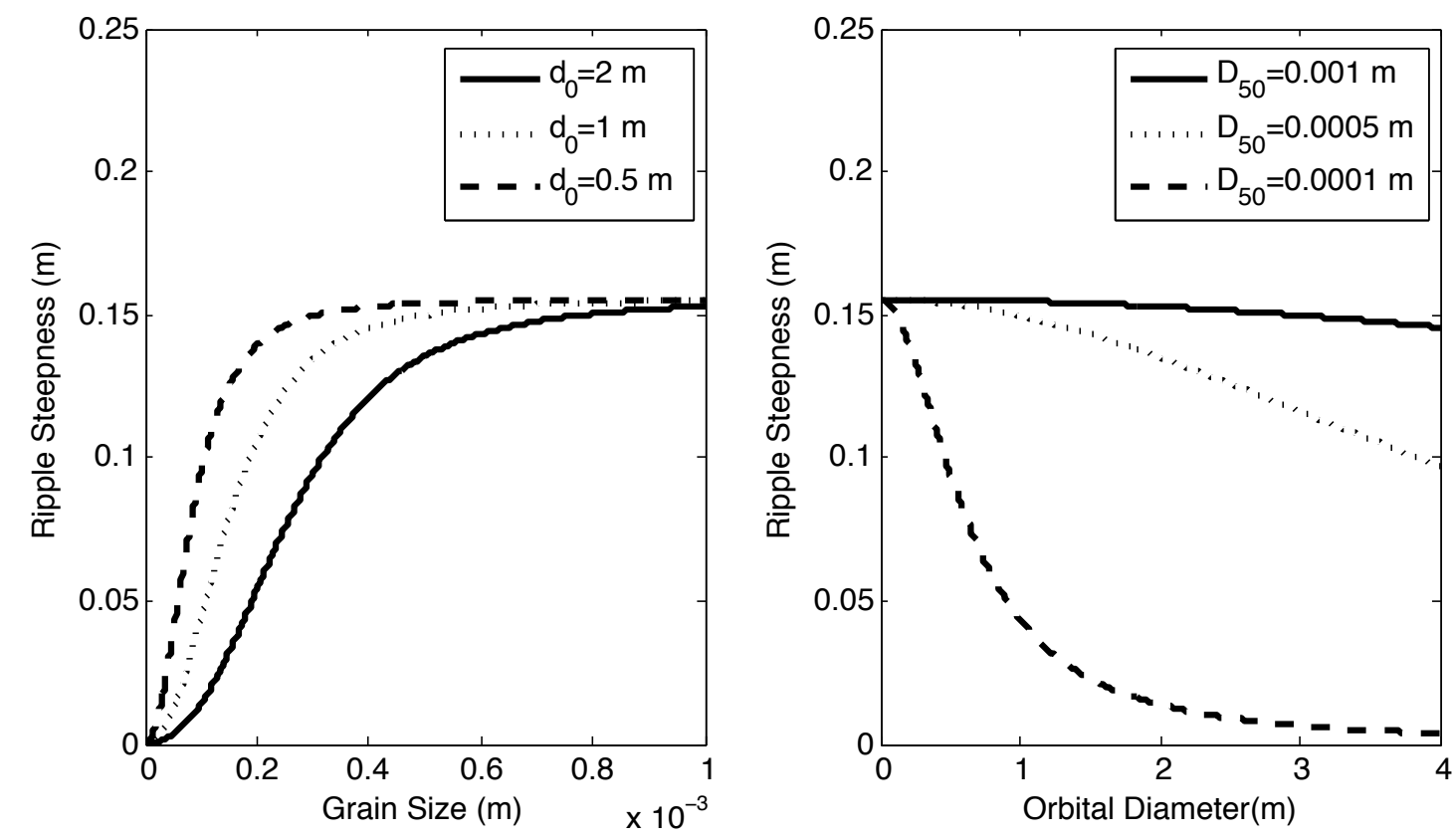

1055 
1056 Figure 14: GP predictor of ripple steepness (13), Predictor based on linear convolution of

1057 GP height and length (11), Soulsby and Whitehouse (2005) predictor (4) and Pedocchi

1058 and García (2009a) predictor (5) and (6) evaluated using only the independent testing

1059 dataset. Top row shows the predictors in linear space, while bottom row shows log-log

1060 space.
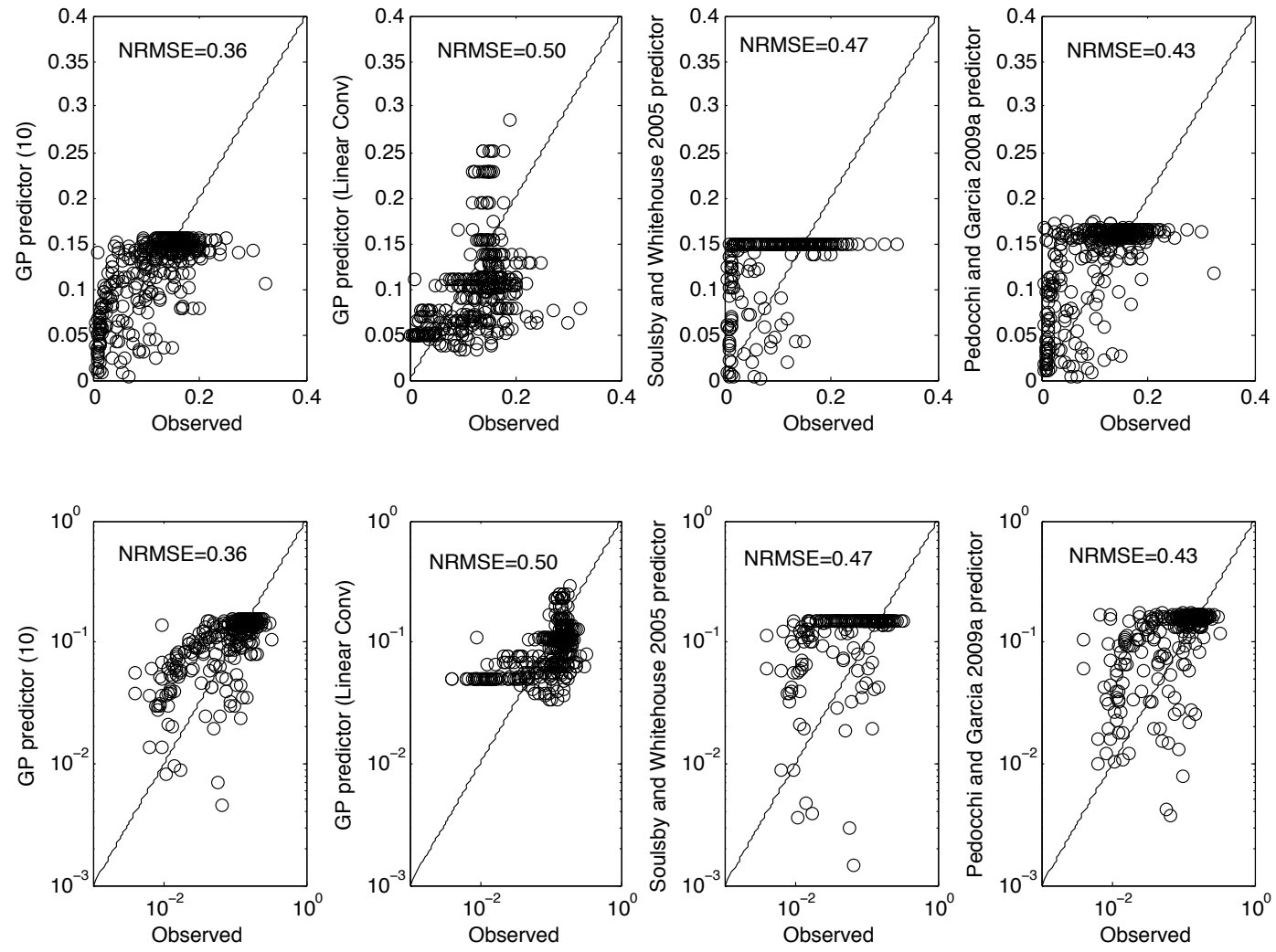

1061 